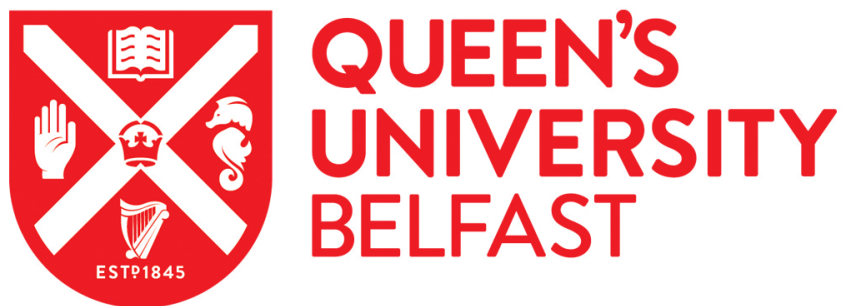

\section{NGTS clusters survey. I. Rotation in the young benchmark open cluster Blanco 1}

Gillen, E., Briegal, J. T., Hodgkin, S. T., Foreman-Mackey, D., Van Leeuwen, F., Jackman, J. A. G., McCormac, J., West, R. G., Queloz, D., Bayliss, D., Goad, M. R., Watson, C. A., Wheatley, P. J., Belardi, C., Burleigh, M. R., Casewell, S. L., Jenkins, J. S., Raynard, L., Smith, A. M. S., ... Vines, J. I. (2020). NGTS clusters survey. I.

Rotation in the young benchmark open cluster Blanco 1. Monthly Notices of the Royal Astronomical Society, 492(1), 1008-1024. https://doi.org/10.1093/mnras/stz3251

Published in:

Monthly Notices of the Royal Astronomical Society

Document Version:

Publisher's PDF, also known as Version of record

Queen's University Belfast - Research Portal:

Link to publication record in Queen's University Belfast Research Portal

Publisher rights

Copyright 2020 The Author(s) Published by Oxford University Press on behalf of the Royal Astronomical Society. This work is made available online in accordance with the publisher's policies. Please refer to any applicable terms of use of the publisher.

\section{General rights}

Copyright for the publications made accessible via the Queen's University Belfast Research Portal is retained by the author(s) and / or other copyright owners and it is a condition of accessing these publications that users recognise and abide by the legal requirements associated with these rights.

Take down policy

The Research Portal is Queen's institutional repository that provides access to Queen's research output. Every effort has been made to ensure that content in the Research Portal does not infringe any person's rights, or applicable UK laws. If you discover content in the Research Portal that you believe breaches copyright or violates any law, please contact openaccess@qub.ac.uk. 


\title{
NGTS clusters survey - I. Rotation in the young benchmark open cluster Blanco 1
}

\author{
Edward Gillen ${ }^{\odot},{ }^{1 \star} \dagger$ Joshua T. Briegal, ${ }^{1}$ Simon T. Hodgkin, ${ }^{2}$ \\ Daniel Foreman-Mackey, ${ }^{3}$ Floor Van Leeuwen, ${ }^{2}$ James A. G. Jackman ${ }^{\oplus}, 4,5$ \\ James McCormac, ${ }^{4,5}$ Richard G. West ${ }^{\oplus}, 4,5$ Didier Queloz, ${ }^{1}$ Daniel Bayliss ${ }^{\oplus}, 4,5$ Michael \\ R. Goad, ${ }^{6}$ Christopher A. Watson, ${ }^{7}$ Peter J. Wheatley ${ }^{\oplus},{ }^{4,5}$ Claudia Belardi, ${ }^{6}$ Matthew \\ R. Burleigh, ${ }^{6}$ Sarah L. Casewell, ${ }^{6}$ James S. Jenkins ${ }^{\oplus,}{ }^{8,9}$ Liam Raynard, ${ }^{6}$ Alexis M. \\ S. Smith, ${ }^{10}$ Rosanna H. Tilbrook ${ }^{6}$ and Jose I. Vines ${ }^{8}$
}

Affiliations are listed at the end of the paper

Accepted 2019 October 3. Received 2019 October 3; in original form 2019 March 7

\begin{abstract}
We determine rotation periods for 127 stars in the $\sim 115$-Myr-old Blanco 1 open cluster using $\sim 200 \mathrm{~d}$ of photometric monitoring with the Next Generation Transit Survey. These stars span F5-M3 spectral types $\left(1.2 \mathrm{M}_{\odot} \gtrsim M \gtrsim 0.3 \mathrm{M}_{\odot}\right)$ and increase the number of known rotation periods in Blanco 1 by a factor of four. We determine rotation periods using three methods: Gaussian process (GP) regression, generalized autocorrelation function (G-ACF), and LombScargle (LS) periodogram, and find that the GP and G-ACF methods are more applicable to evolving spot modulation patterns. Between mid-F and mid-K spectral types, single stars follow a well-defined rotation sequence from $\sim 2$ to $10 \mathrm{~d}$, whereas stars in photometric multiple systems typically rotate faster. This may suggest that the presence of a moderate-to-high mass ratio companion inhibits angular momentum loss mechanisms during the early pre-main sequence, and this signature has not been erased at $\sim 100$ Myr. The majority of mid-F to mid$\mathrm{K}$ stars display evolving modulation patterns, whereas most $\mathrm{M}$ stars show stable modulation signals. This morphological change coincides with the shift from a well-defined rotation sequence (mid-F to mid-K stars) to a broad rotation period distribution (late-K and $\mathrm{M}$ stars). Finally, we compare our rotation results for Blanco 1 to the similarly aged Pleiades: the single-star populations in both clusters possess consistent rotation period distributions, which suggests that the angular momentum evolution of stars follows a well-defined pathway that is, at least for mid-F to mid-K stars, strongly imprinted by $\sim 100$ Myr.
\end{abstract}

Key words: stars: rotation-stars: variables: general-binaries: general-open clusters and associations: individual: Blanco 1.

\section{INTRODUCTION}

The initial mass, composition, and angular momentum of a star define much of its evolutionary pathway. Rotation influences the internal structure, mixing, and energy transport in stars, as well as driving the stellar dynamo, which in turn gives rise to star-spots, high-energy radiation, and stellar winds (Bouvier et al. 2014).

At the start of the pre-main sequence (PMS), solar-type and lowmass stars $\left(M>0.3 \mathrm{M}_{\odot}\right)$ typically have rotation periods between 1 and $10 \mathrm{~d}$ and show a bimodal rotation distribution with peaks at

^E-mail: ecg41@ cam.ac.uk

$\dagger$ Winton Fellow. $\sim 2$ and $8 \mathrm{~d}$ (Herbst, Bailer-Jones \& Mundt 2001). This bimodality is usually attributed to the presence of circumstellar discs, with the slow rotators thought to be prevented from spinning up due to ongoing interaction with their discs, whereas the fast rotators are believed to have already dissipated their inner discs and hence are spinning up towards the zero-age main sequence (ZAMS; Barnes 2003; Bouvier 2013). Rotation rates increase towards the ZAMS, where stars of a given mass arrive with a range of rotation velocities (e.g. Stauffer \& Hartmann 1987; Terndrup et al. 2000; Rebull et al. 2016a, b).

During the main sequence (MS) stage of evolution, rotation rates decrease and stars of a given mass converge to rotate with a characteristic period that increases with time. The time for this convergence is mass dependent: for stars with convective envelopes, 
higher mass stars converge faster than their lower mass counterparts (Stauffer, Hartmann \& Latham 1987). During this evolution, angular momentum is lost through magnetized stellar winds (e.g. Chaboyer, Demarque \& Pinsonneault 1995a, b; Reiners \& Mohanty 2012) and redistributed within the stellar interior (e.g. Eggenberger, Maeder \& Meynet 2005; Lagarde et al. 2012; Charbonnel et al. 2013), which leaves older stars with weaker magnetic fields and lower levels of high-energy radiation (e.g. Vidotto et al. 2014; Johnstone et al. 2015, and references therein). Recent theoretical models now reproduce the main PMS and MS evolutionary trends in the observed rotation behaviour of solar-type and low-mass stars (Gallet \& Bouvier 2013, 2015).

Young open clusters offer a particularly useful tool for understanding the evolution of stellar rotation during the first billion years $(t<1 \mathrm{Gyr})$. Open clusters are populations of stars that span a range of masses but possess essentially the same age and composition. Observational studies of rotation in young open clusters date back to at least the 1960 s, but rapidly expanded as large-scale photometric surveys arose using wide-field cameras on small-to-medium class telescopes, e.g. the Monitor Survey (Irwin et al. 2006), HATNet (Hartman et al. 2010), SuperWASP (Delorme et al. 2011), KELT (Cargile et al. 2014), PTF (Covey et al. 2016a), and K2 (Rebull et al. 2016a, b, 2017; Stauffer et al. 2016; Douglas et al. 2017, 2019). ${ }^{1}$

Rotation periods can be determined from photometric monitoring of young active stars by tracking the brightness modulation patterns that arise from the longitudinal inhomogeneity of surface starspot distributions as the stars rotate. In principle, this is relatively straightforward, but complications arise from the fact that starspots appear, evolve in both size and position, and disappear over many rotation periods. The complexity of methods used to estimate rotation periods from stellar light curves therefore should be matched to the precision and duration of the data analysed.

Combining rotation period information from clusters of different ages allows us to probe the evolution of stellar rotation as a function of age. Pairs of similarly aged clusters are particularly valuable, as they offer a means to determine the rotation period distribution at a given age from two independent samples of stars in different cluster environments. Such pairs are rare, especially at older $(t>100 \mathrm{Myr})$ ages, with the two main examples being the Hyades and Praesepe (both 700-800 Myr; Brandt \& Huang 2015a, b), and the Pleiades and Blanco 1 (both 100-120 Myr; Gaia Collaboration 2018, hereafter B18). ${ }^{2}$ The Pleiades, Hyades, and Praesepe all have a long history of rotation studies, culminating in recent observations by Kepler/K2 spanning $75 \mathrm{~d}$. Blanco 1, however, lacks such a precise longterm photometric monitoring campaign. This motivated the Next Generation Transit Survey (NGTS; Chazelas et al. 2012; Wheatley et al. 2018) to observe Blanco 1 with precise mmag photometry spanning $\sim 200 \mathrm{~d}$; these observations form the basis of this paper.

Blanco 1 is a $\sim 115$-Myr-old Galactic open cluster, situated in the local spiral arm at a distance of $\sim 240 \mathrm{pc}$ in the direction towards and below the Galactic Centre (B18). It is comprised of 489 Gaia DR2-confirmed stars, ranging from $\mathrm{B}$ to $\mathrm{M}$ spectral types, as well as tens of likely brown dwarf members down to $\sim 30 M_{\mathrm{J}}$ (Moraux et al.

\footnotetext{
${ }^{1}$ HATNet $=$ Hungarian-made Automated Telescope Network; SuperWASP $=$ Super Wide Angle Search for Planets; KELT $=$ the Kilodegree Extremely Little Telescope; PTF = Palomar Transient Factory; $K 2=$ Kepler $/ K 2$.

${ }^{2}$ We note that M35 and NGC 2516 have traditionally both been assigned ages of $\sim 150 \mathrm{Myr}$ (Irwin et al. 2007; Meibom, Mathieu \& Stassun 2009), but the Gaia DR2 estimated age of NGC 2516 from its Hertzsprung-Russell diagram in B18 is 2300 Myr. Without a comparable DR2-derived age for M35 in B18, we refrain from labelling them as similarly aged here.
}

2007; Casewell et al. 2012; B18). The cluster has a solar metallicity ([Fe/H] 0.03; Netopil et al. 2016), an on-sky stellar density of $\sim 30$ stars $\mathrm{pc}^{-2}$ (Moraux et al. 2007), and a low reddening along the line of sight $(E(B-V) \sim 0.010 ; \mathrm{B} 18)$. Given these properties, Blanco 1 is much like a scaled-down version of the Pleiades $(\sim 110 \mathrm{Myr}$, 1326 Gaia DR2 members, on-sky stellar density $\sim 65 \mathrm{stars} \mathrm{pc}^{-2}$, $[\mathrm{Fe} / \mathrm{H}] \sim-0.01$; Moraux et al. 2003; B18). Its main outstanding property is its high Galactic latitude $\left(b=-79^{\circ}\right)$, especially given its moderate proper motion and UVW velocities, which hint at an unusual formation/evolution history compared to most young open clusters (normally located close to the Galactic plane).

Blanco 1 was first noted by Blanco (1949) and has been extensively studied over the last $70 \mathrm{yr}$. Initial photometric observations identified many of the higher mass members (e.g. Westerlund 1963; Epstein 1968; de Epstein \& Epstein 1985; Westerlund et al. 1988), with subsequent photometric and astrometric studies revealing the lower mass population (e.g. Moraux et al. 2007; Platais et al. 2011; Casewell et al. 2012). Spectroscopic studies of Blanco 1 have broadly characterized the radial velocity (RV) distribution of the cluster (e.g. Mermilliod et al. 2008; González \& Levato 2009; Mermilliod, Mayor \& Udry 2009) and provided $v \sin i$ measurements of the brighter moderate-to-rapid rotators in the cluster. The rotation of stars in Blanco 1 has been studied by Cargile et al. (2014), who report photometric rotation periods for 33 stars with spectral types between late-A/early-F and mid-K. Blanco 1 has also been the subject of X-ray surveys (Micela et al. 1999; Pillitteri et al. 2003, 2004, 2005), as well as searches for debris discs (Stauffer et al. 2010) and stellar flares (Leitzinger et al. 2014). Most recently, Gaia DR2 produced a homogeneous membership list for Blanco 1 spanning the cluster's full stellar sequence (B18); we use this Gaia DR2 membership list in this study.

This paper presents a study of rotation in Blanco 1 using $\sim 200 \mathrm{~d}$ of ground-based data from NGTS. We introduce the NGTS observations in Section 2. In Section 3, we estimate rotation periods using three methods: Gaussian process (GP) regression, generalized autocorrelation function (G-ACF), and Lomb-Scargle (LS) periodogram, and then compare their predictions. In Section 4, we identify likely multiple-star systems using colour-magnitude diagrams (CMDs). We then discuss our rotation periods for Blanco 1 in Section 5, before comparing to the Pleiades in Section 6. We conclude in Section 7.

\section{NGTS OBSERVATIONS OF BLANCO 1}

NGTS comprises twelve 20-cm wide-field roboticized telescopes situated at the ESO Paranal Observatory in Chile. The facility is optimized to detect small exoplanets orbiting $\mathrm{K}$ and early $\mathrm{M}$ stars (e.g. Bayliss et al. 2018; West et al. 2019), and is designed to achieve mmag photometric precision across each camera's $2.8^{\circ}$ field of view (FoV).

Blanco 1 was observed using a single NGTS camera over a 195night baseline between 2017 May 7 and November 18. 201773 exposures were obtained, at $13 \mathrm{~s}$ cadence (with $10 \mathrm{~s}$ exposures), on 134 nights within this period. Of the 489 Blanco 1 members from B18, the NGTS FoV encompassed 429 stars (88 percent of the cluster members). 170 of these stars had an apparent magnitude brighter than $16 \mathrm{mag}$ in the NGTS band, and therefore had photometry automatically extracted by the NGTS pipeline, before being binned to 30 min cadence for this work. The NGTS band covers the $520-890 \mathrm{~nm}$ range, and is therefore similar to a combined $R+I$ filter. We refer the interested reader to Wheatley et al. (2018) for further details on the NGTS filter and pipeline. 


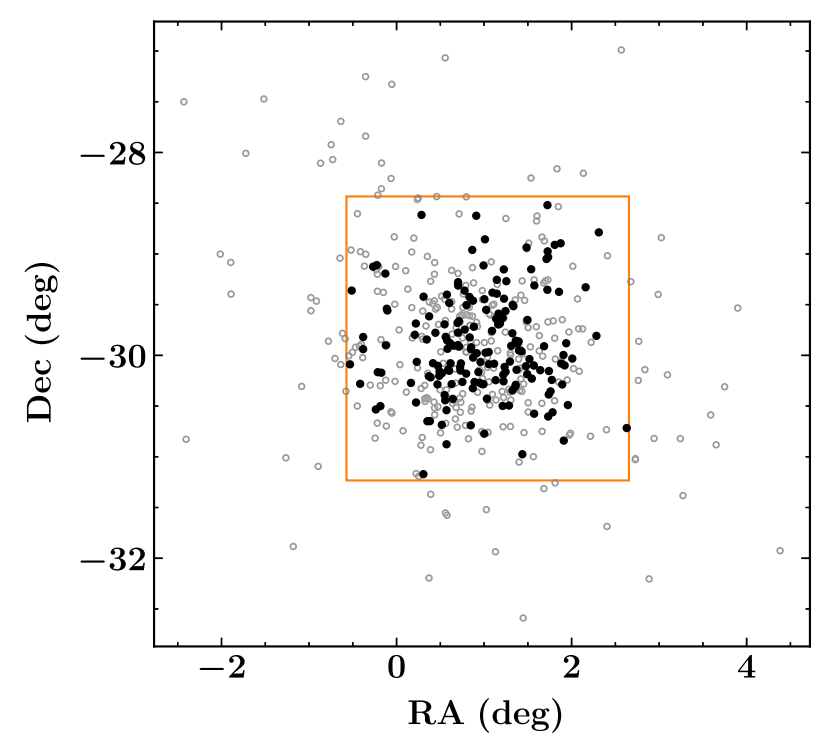

Figure 1. Spatial distribution of Blanco 1 members from B18. The orange box indicates the NGTS FoV, filled black circles represent stars with NGTS light curves, and open grey circles indicate stars that either were too faint or fell outside the NGTS FoV.

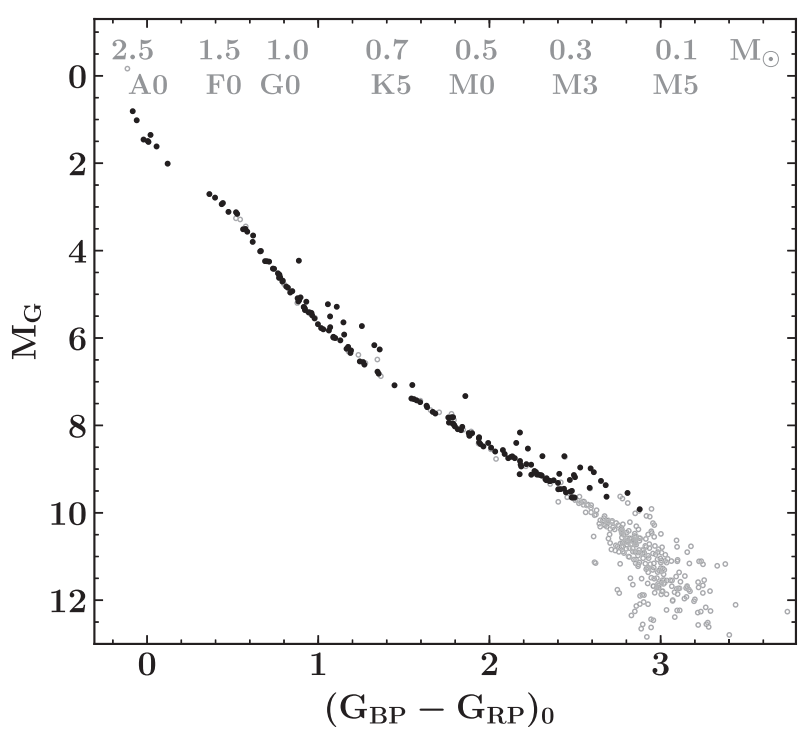

Figure 2. Absolute Gaia $\mathrm{G}$ versus $G_{\mathrm{BP}}-G_{\mathrm{RP}} \mathrm{CMD}$ for members of Blanco 1 from B18 (open grey circles) highlighting stars observed by NGTS (filled black circles). The Gaia photometry and parallaxes reveal a tight cluster sequence with a scattering of likely multiple-star systems lying above the single-star sequence. For reference, an equal-mass binary produces a 0.75 mag excess. The $G_{\mathrm{BP}}-G_{\mathrm{RP}}$ colours have been dereddened assuming $E(B$ $-V)=0.010$ for the cluster (B18). NGTS observed essentially all cluster members down to a spectral type of $\sim \mathrm{M} 3$. The stellar masses are MIST model predictions (Choi et al. 2016; Dotter 2016) evaluated at the age of Blanco 1, and the spectral types were estimated using updated information from Pecaut \& Mamajek (2013) (E. Mamajek online table; see text).

Figs 1 and 2 show the spatial and colour-magnitude distributions of Blanco 1 members, highlighting stars with NGTS light curves. Given the mass segregation within the cluster and NGTS's brightness range, nearly all stars in the cluster down to a spectral type of $\sim \mathrm{M} 3$ have NGTS light curves. The spectral types in Fig. 2 (as well as other figures and tables presented here) were estimated using updated information from Pecaut \& Mamajek (2013) ${ }^{3}$ based on their intrinsic $G-K_{\mathrm{s}}$ and $G_{\mathrm{BP}}-G_{\mathrm{RP}}$ colours, i.e. $\left(G-K_{\mathrm{s}}\right)_{0}$ and $\left(G_{\mathrm{BP}}-G_{\mathrm{RP}}\right)_{0}$.

\section{ESTIMATING ROTATION PERIODS}

We test three methods: LS periodogram, G-ACF, and GP regression. These differ in their assumptions and complexity, and hence in their appropriateness for estimating rotation periods from photometric rotational modulation. We introduce each of these methods below (Sections 3.1, 3.2, and 3.3) before comparing their assumptions and predictions in Section 3.4.

\subsection{Lomb-Scargle periodogram}

The LS periodogram is a standard method for detecting periodic signals in unevenly sampled data (see VanderPlas 2018, for a detailed discussion). It has been widely used to estimate stellar rotation periods from stars in the field (e.g. Nielsen et al. 2013) as well as open clusters (e.g. Cargile et al. 2014; Rebull et al. 2016a). LS models the observed variability as a sine wave with a given period. As discussed in VanderPlas (2018), LS is the optimal statistic for fitting a sinusoid to data, but this is not the same as being optimally suited to finding the period of a generic sinusoidal-like signal, such as photometric modulation arising from the rotation of spotted stars. For a light curve displaying photometric modulation, LS determines the best-fitting sinusoid, which makes the implicit assumption that both the modulation period and its phase shape (i.e. shape within a given rotation cycle) are constant in time. This is usually an acceptable assumption for low-mass stars with stable modulation patterns, but not for many solar-type stars, which display evolving signals (see Sections 3.4.2 and 5.2 for further discussion).

\subsection{Generalized autocorrelation function}

Autocorrelation, i.e. correlating a data set with itself, is a welltested 'model-free' approach to estimating rotation periods from stellar light curves (see e.g. McQuillan, Mazeh \& Aigrain 2014, for application to Kepler data). Periodogram methods generally assume a sinusoidal basis function, which can lead to incorrect solutions when presented with non-sinusoidal signals. The model-free ACF therefore is applicable to all light curves, irrespective of the shape and evolution of the variability signal.

Traditional autocorrelation is limited to regularly sampled time series (typically space-based monitoring data), which has been a limiting factor in its wider application to ground-based (and other non-regularly sampled) data sets. Here, we use the new G-ACF, which is a generalized version of the standard ACF that is applicable to both regularly and irregularly sampled time series. The algorithm is introduced in Kreutzer et al. (in preparation) and described in detail in Briegal et al. (in preparation), with specific application to extracting stellar rotation periods for field stars with NGTS. We therefore give only a brief description of the algorithm below and refer the interested reader to these publications.

Performing ACF on irregularly sampled data is possible if we (i) generalize the ACF 'lag' term from an integer multiple of the sampling constant to a real parameter and (ii) define selection and weight functions to decide how to identify and interpret correlations

${ }^{3}$ http://www.pas.rochester.edu/ emamajek/EEM_dwarf_UBVIJHK_colors_ Teff.txt 
between data points whose timestamps do not perfectly align for a given lag (time shift). For each data point, and at each lag, we choose the closest unshifted data point in time to correlate with and weight the correlation between each pair of data points by a function that is inversely proportional to their time difference.

We estimate rotation periods from the ACF by calculating a twostage fast Fourier transform (FFT). First, we calculate the FFT of the whole ACF to identify approximate periods, and then refine these by using only the section of the ACF within up to five times the identified period. Calculating FFT on the ACF is more appropriate than on the light curves themselves, as the ACF is more sinusoidal than the stellar modulation patterns in most cases. We refer the reader to Briegal et al. (in preparation) for further details on this procedure. In this work, the primary reason for this two-stage process was to account for evolution present in the rotation signals of solar-type stars (see Section 3.4 for further discussion).

\subsection{Gaussian process regression}

It has been demonstrated that a GP can be used as a descriptive model to measure stellar rotation periods (Angus et al. 2018). A GP is a model for the covariance between data points or, in other words, the autocorrelation of the time series. This means that we can use a GP with a quasi-periodic covariance and interpret the parameters of that model as physical properties of the time series. We extend the framework presented by Angus et al. (2018) to include scalable computation of the GP model using the CELERITE algorithm (Foreman-Mackey et al. 2017) and we improve the runtime of the inference procedure by taking advantage of a scalable method for computing the gradient of CELERITE models with respect to their parameters (Foreman-Mackey 2018). This efficient calculation of gradients enables the use of the efficient noU-turn sampling (Hoffman \& Gelman 2014) method for posterior inference. This fitting procedure is implemented as part of the EXOPLANET project (Foreman-Mackey \& Barentsen 2019) and is built on top of THEANO (The Theano Development Team 2016) for efficient model evaluation, PYMC3 (Salvatier, Wiecki \& Fonnesbeck 2016) for inference, and ASTROPY (Astropy Collaboration 2013, 2018) for data manipulation.

The kernel function that we use to model the covariance caused by stellar variability is a mixture of three stochastically driven damped simple harmonic oscillators (SHOs). This function is described in more detail by Foreman-Mackey et al. (2017), but each oscillator introduces one term into the description of the kernel and each term $k$ has the power spectrum

$S_{k}(\omega)=\sqrt{\frac{2}{\pi}} \frac{S_{0, k} \omega_{0, k}^{4}}{\left(\omega^{2}-\omega_{0, k}^{2}\right)^{2}+\omega_{0, k}^{2} \omega^{2} / Q_{k}^{2}}$,

where $\omega$ is the angular frequency, $S_{0, k}$ is the amplitude of the oscillation, $\omega_{0, k}$ is the undamped frequency, and $Q_{k}$ is the quality factor.

We fix $Q_{1}=1 / \sqrt{2}$ so that the first term can capture any nonperiodic covariance in the time series (Foreman-Mackey et al. 2017) and the other two terms are constrained to have frequencies that differ by a factor of 2 . Specifically, we define the parameters of the second and third terms as follows:

$Q_{2}=Q_{3}+\Delta Q$,

$\omega_{0,2}=\frac{4 \pi Q_{2}}{P_{\text {rot }} \sqrt{4 Q_{2}^{2}-1}}$,
$S_{0,2}=\frac{A}{\omega_{0,2} Q_{2}}$,

$\omega_{0,3}=\frac{8 \pi Q_{3}}{P_{\text {rot }} \sqrt{4 Q_{3}^{2}-1}}$,

$S_{0,3}=\frac{f A}{\omega_{0,3} Q_{3}}$,

parametrized by a rotation period $P_{\text {rot }}$, the quality factor of the third term $Q_{3}>1 / 2$, the difference between the quality factor of the second and third terms $\Delta Q>0$, the amplitude of the base harmonic $A>0$, and the fractional amplitude of the third term $0<f<1$.

We initialize the sampler using the rotation period estimate from either $\mathrm{LS}$ or $\mathrm{G}-\mathrm{ACF}{ }^{4}$ perform an initial maximum a posteriori (MAP) fit, identify $3 \sigma$ outliers from this initial solution, and then mask the outliers and refit. Masking these outliers was designed primarily to remove flares, but also other non-rotation phenomena such as eclipses. For most stars, around 10 data points were flagged as outliers and masked, although flaring stars typically had more. We then run four independent Markov chains for 2000 steps each to tune the mass matrix of the proposal, followed by four production chains of 2000 steps each. This procedure takes about $10 \mathrm{~min}$ to run using two CPUs and generally results in several thousand effective samples of the rotation period that we use to approximate the posterior probability density.

\subsection{Comparison between LS, G-ACF, and GPs}

\subsubsection{Assumptions underlying the three methods}

The three methods differ in their assumptions and flexibility, which can be seen in their predictions for the rotation periods of stars displaying different light-curve morphologies. Fig. 3 shows two examples of light curves that display regular modulation patterns, which do not significantly evolve during the 200-d light curves. LS, G-ACF, and GPs all typically predict consistent rotation periods for such stars. Fig. 4 shows two examples of light curves whose modulation patterns evolve significantly during the 200-d NGTS observations. For these two stars, GPs and G-ACF agree to within $1 \sigma$, but the LS period is discrepant by $>5 \sigma$ (compared to the GP posterior period distribution). The light curves and period results for all stars with detected rotation periods are given in the supplementary material in the online journal. The two sets of examples in Figs 3 and 4 highlight some of the assumptions underlying the three methods:

(i) LS is essentially a rigid sine-wave model and is therefore best suited to light curves whose modulation patterns are purely sinusoidal and do not evolve throughout the observations. It is less well suited to determining rotation periods from stellar light curves whose modulation signal (primarily the phase shape) evolves appreciably during the observations, for example from evolving active regions and/or differential rotation.

(ii) G-ACF on the other hand, as the data themselves are the model, is suitable for extracting periods from light curves irrespective of the modulation shape and evolutionary time-scale. Complications from this method arise due to the diurnal nature of the observations, which give rise to a low-level 1-d signal imparted on the ACF that can subtly modify the exact shape of ACF peaks and hence the estimated rotation periods.

\footnotetext{
${ }^{4}$ We note that the exact initial guess is not important as long as it is reasonably close to the actual rotation period.
} 

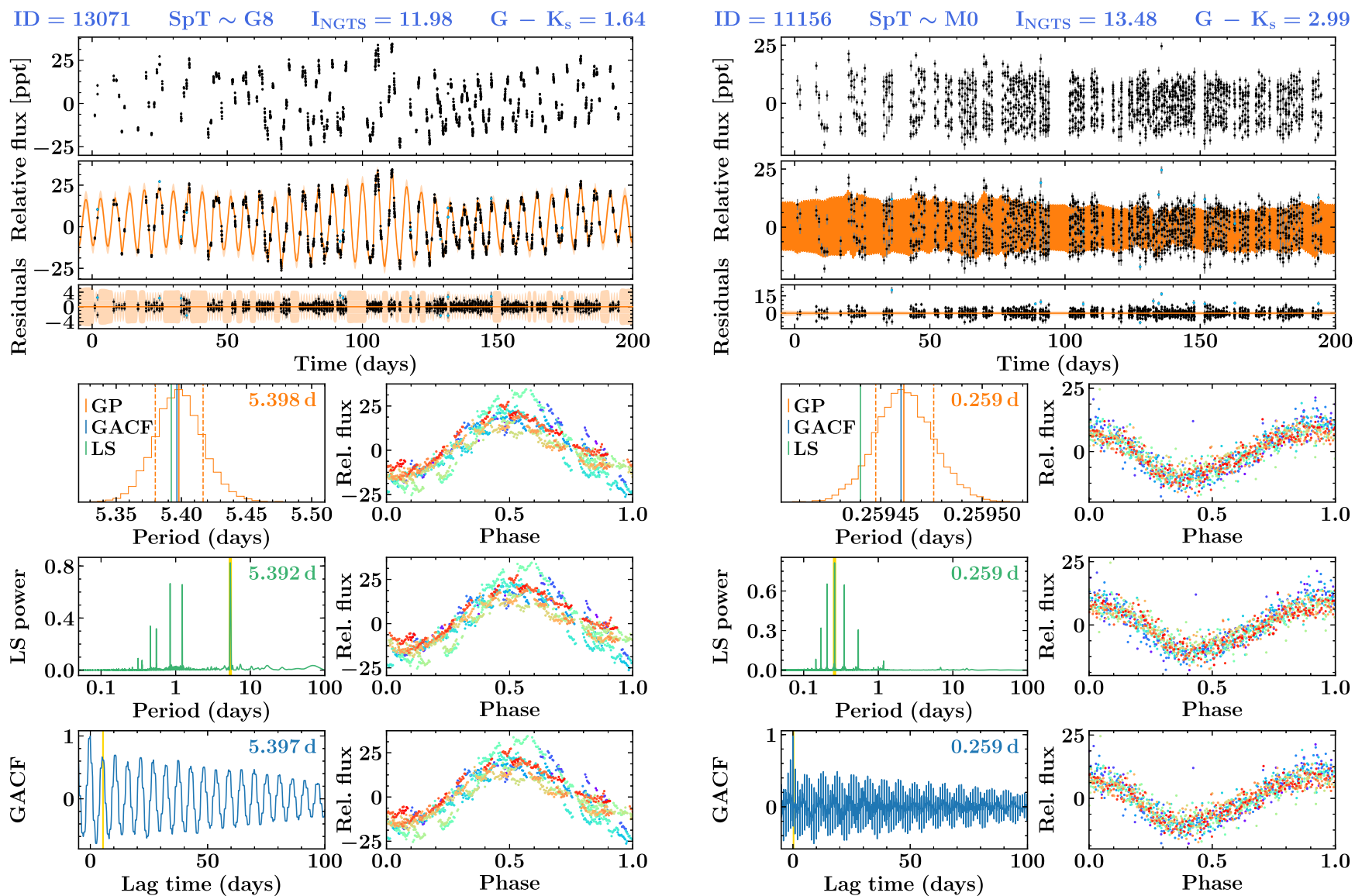

Figure 3. NGTS light curves and period predictions using GPs, LS, and G-ACF, for two stars (object IDs 13071 and 11156 , left and right, respectively). In each case, the top three plots show the relative flux NGTS light curve in units of parts per thousand (ppt; top), the NGTS light curve with the MAP GP model (middle), and residuals (bottom). The orange line and shaded region show the mean and $1 \sigma$ uncertainty on the MAP GP model. In the residuals plot, blue points indicate outlying data that were masked by the GP during the fit. The bottom six plots show the period estimation results (left column) for GPs (top), LS (middle), and G-ACF (bottom), along with the NGTS data phase-folded on each method's period (right column). Top left: 1D GP posterior period distribution (orange) with the median period and $1 \sigma$ uncertainties (solid and dashed orange lines) shown and the period printed top right. For comparison, the period predictions of G-ACF and LS are shown by the vertical blue and green solid lines, respectively. Middle left: LS periodogram in green with the identified period highlighted in yellow and printed top right. Bottom left: G-ACF in blue (positive direction shown only) with the identified period highlighted in yellow and printed top right. Right column: NGTS light curve phase-folded on the corresponding period (GP, LS, and G-ACF, top to bottom), with the rainbow colour scheme indicating data from the beginning (indigo) to the end (red) of the observations. The modulation patterns of these two stars are relatively stable during the $200 \mathrm{~d}$ of observations and hence the period predictions from GPs, LS, and G-ACF all agree.

(iii) GPs lie somewhere between LS and G-ACF in terms of their assumptions. They form a class of model, akin to LS, that depends on both the covariance kernel chosen and the covariance properties of the data being analysed. This drawing of information from the data themselves shares some similarity with the principles underlying the G-ACF method. What sets the GP method apart, in the current context, is that by drawing covariance information from the data and interpreting it through a kernel to generate a model, the GP has predictive power. This predictive power can be seen between individual nights and before and after the observations (see the second-from-top panels in Figs 3 and 4). Furthermore, based on tests where subsections of light curves were masked, the GP models were able to adequately predict the stellar modulation patterns over a few-to-several rotation periods, depending on the level of evolution present in the light curve.

Underlying all three methods is a general assumption that the light-curve modulation patterns have a single underlying period. Multiple periods can be detected, however: in LS and G-ACF, from two or more unrelated peaks, and for GPs by double-peaked period distributions (if sufficiently close that the Markov chains explore that part of parameter space). Of course, to correctly estimate individual periods from a single light curve when multiple periodic signals are present, either due to differential rotation or due to the system being a multiple-star system, a composite model needs to be applied, which is not the case here. Therefore, we make no attempt to try and characterize differential rotation in our light curves or characterize the rotation periods of individual stars in identified multiple-star systems. We refer the reader to Gillen et al. (2017) as an example of such an effort, but leave this to future work here.

\subsubsection{Comparison across the Blanco 1 sample}

Of the 170 Blanco 1 stars observed by NGTS, we detected rotation periods in 127 stars. For 118 of these, all three methods detected the same modulation signal, but for the remaining 9 stars either aliases or different signals were preferred by one or more methods. 

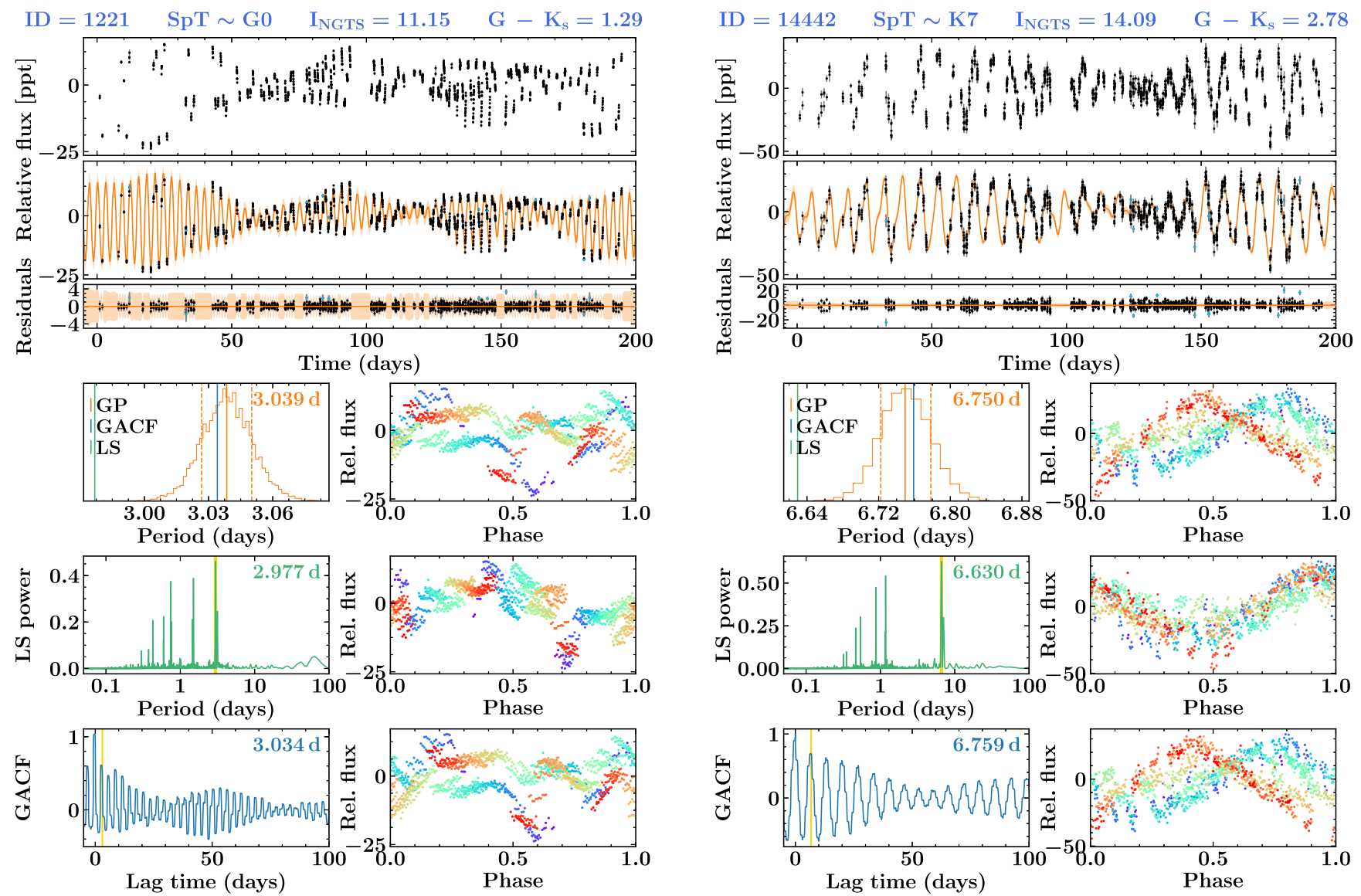

Figure 4. Same as Fig. 3, but for objects 1221 and 14442, two stars whose modulation patterns evolve significantly during the 200-d observations. The GP and G-ACF predictions agree well for these stars, given that they can account for the evolution in the modulation pattern, but the LS prediction is offset because it finds the period that is best fit by a non-evolving sine wave, which is not appropriate for these stars.

In Fig. 5, we compare the agreement between the periods extracted by our three methods using their fractional period differences, i.e. $\left[\left(P_{1}-P_{2}\right) / P_{1}\right] \times 100$ per cent, as a function of $(G-$ $\left.K_{\mathrm{s}}\right)_{0}$ colour. We opted to compare against $\left(G-K_{\mathrm{s}}\right)_{0}$ colour rather than rotation period because it is a more fundamental parameter (being a proxy for mass) and better separates different light-curve morphologies (see Section 5.2). Essentially, mid-F to mid-K stars $\left(\left(G-K_{\mathrm{S}}\right)_{0} \lesssim 2.5\right)$ typically show evolution in their modulation patterns (both in amplitude and in phase shape), whereas late-K and especially $\mathrm{M}$ stars $\left(\left(G-K_{\mathrm{s}}\right)_{0} \gtrsim 2.5\right)$ generally display more stable sinusoidal modulation. The evolutionary nature of the light curves is the main cause of disagreement between methods. We note that the faintest stars in our sample sometimes contained residual moon variations arising from incomplete background correction, which we fit and removed as described in Appendix A. For these stars, the three methods typically agree well.

The GP and G-ACF periods (cyan) agree best, as they are the two most flexible methods, whose agreement is consistently within 2-3 percent across the whole Blanco 1 sample. Both G-ACF versus LS (magenta) and GP versus LS (black) show the same general trend: the agreement is good for late-K and M stars $((G-$ $\left.\left.K_{\mathrm{s}}\right)_{0} \gtrsim 2.5\right)$, whose modulation patterns are stable and sinusoidal, but are noticeably worse for the mid-F to mid-K stars $((G-$ $\left.K_{\mathrm{s}}\right)_{0} \lesssim 2.5$ ), which display evolving modulation patterns. The worse agreement for the mid-F to mid-K stars is primarily due to the rigid nature of the LS algorithm, which does not allow for any evolution, and essentially finds the period that folds the data closest to a sine wave. GPs and G-ACF, on the other hand, are flexible enough to account for the evolution seen in the Blanco 1 light curves.

We find that GPs and G-ACF perform best across the Blanco 1 sample (F5 to M3 stars). It is worth noting that GPs require an initial guess for the rotation period, whereas G-ACF does not. We therefore see G-ACF as an efficient method for detecting rotation periods from large samples of stars that display a range of modulation morphologies, and GPs as a powerful tool to refine period estimates and to better understand modulation signals.

We visually inspected the GP, LS, and G-ACF results for all light curves. We found that the GP method gave the most reliable rotation periods across the full sample and hence selected the GP periods for all but four stars (where we favoured either the G-ACF or LS period). These four stars possess short $(\lesssim 1 \mathrm{~d})$ periods and stable modulation. We note that the difference between the G-ACF or LS period and the GP period for these stars was $\lesssim 15 \min (0.01 \mathrm{~d})$, so our choosing the G-ACF or LS periods for these stars was to present the best periods we could, rather than the GP period being incorrect per se. As we did not compute errors for the G-ACF and LS periods, we note that these four stars do not have errors associated with their favoured periods but, based on the range of periods resulting in well-defined phase-folded modulation signals, they should all have errors of the order of $\sim 0.02 \mathrm{~d}$ or less. Table 1 reports period information for all stars detected to be periodic, and includes the GP, $\mathrm{G}-\mathrm{ACF}$, and LS periods for all stars, along with the method adopted. 


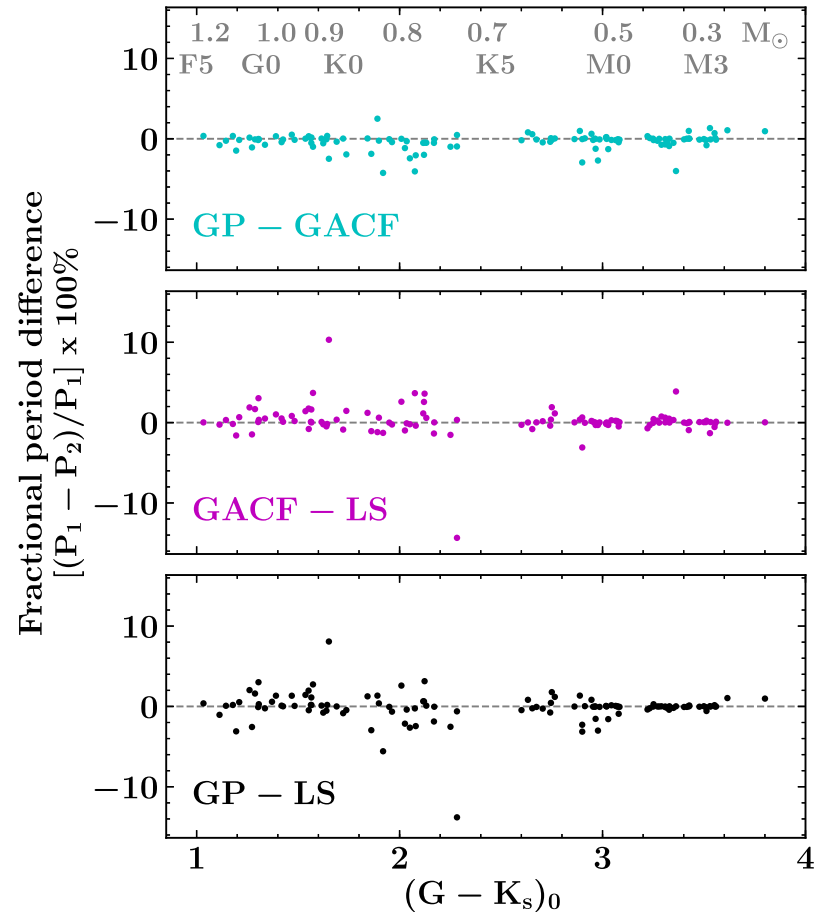

Figure 5. Comparison of the periods extracted for Blanco 1 stars using GP, G-ACF, and LS methods. We compare the period differences between each method (defined as $\left[\left(P_{1}-P_{2}\right) / P_{1}\right] \times 100$ per cent) as a function of dereddened $G-K_{\mathrm{s}}$ colour. For this comparison, we use 118 stars where each method detected the same rotation signal. While the agreement is good for most stars, there are exceptions where different methods disagree by up to $\sim 15$ percent. The agreement is better for the lower mass stars $((G$ $\left.-K_{\mathrm{S}}\right)_{0} \gtrsim 2.5$ ), which typically show stable modulation signals. Solar-type stars $\left(\left(G-K_{\mathrm{s}}\right)_{0} \lesssim 2.5\right)$ display greater evolution in their light curves, which causes a larger scatter in the period estimates between the three methods. Overall, the GP and G-ACF methods (cyan) agree best, most notably for the evolving solar-type members, followed by G-ACF and LS (magenta) and then GP and LS (black).

Additional information, including positions, magnitudes, colours, estimated spectral types, and detected multiplicity (see Section 4), is also given. Table 2 reports the same set of additional information for stars not detected to be periodic.

\subsection{Comparison with literature rotation periods for Blanco 1}

We compare our rotation periods to literature values from Cargile et al. (2014, hereafter C14) as a further sanity check for the novel GP and G-ACF techniques presented here. C14 estimated rotation periods for bright Blanco 1 members using KELT-South light curves, which comprised 43 nights of data spread over 90 nights. Fig. 6 shows the match between our rotation periods and theirs for 23 stars that have detected periods in both surveys and are DR2-confirmed members of the cluster.

18 of the 23 stars (78 per cent) have rotation periods that agree to 10 percent or better, with 17 of these agreeing to within their $1 \sigma$ uncertainties. Of the other five stars, one (NGTS ID $12805^{5}$ ) has a period that agrees to within 20 per cent, which suggests that the same rotation signal is being probed, but the detected period is probably affected by correlated noise in one or both data sets;

\footnotetext{
${ }^{5}$ See online supplementary figures.
}

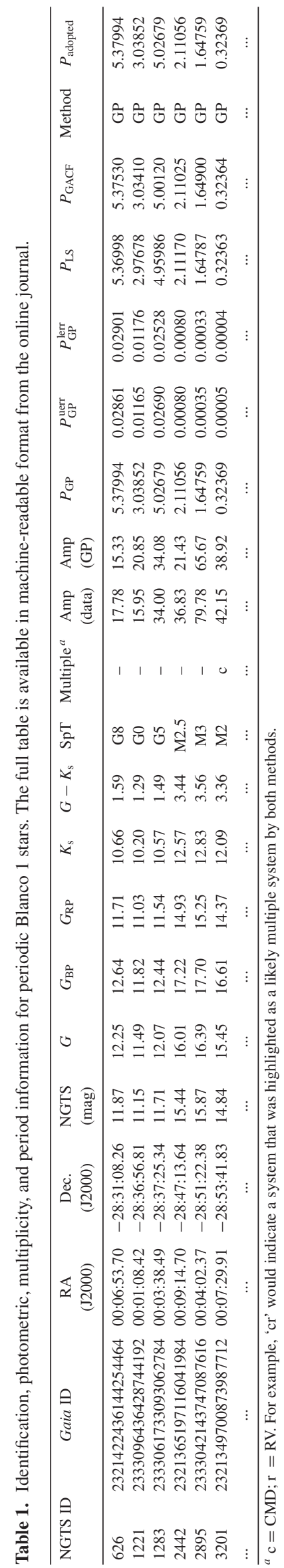


Table 2. Identification, photometric, and multiplicity information for Blanco 1 stars without a detected period. The full table is available in machine-readable format from the online journal.

\begin{tabular}{|c|c|c|c|c|c|c|c|c|c|c|c|}
\hline NGTS ID & Gaia ID & $\begin{array}{c}\text { RA } \\
(\mathrm{J} 2000)\end{array}$ & $\begin{array}{l}\text { Dec. } \\
\text { (J2000) }\end{array}$ & $\begin{array}{c}\text { NGTS } \\
\text { (mag) }\end{array}$ & $G$ & $G_{\mathrm{BP}}$ & $G_{\mathrm{RP}}$ & $K_{\mathrm{s}}$ & $G-K_{\mathrm{s}}$ & $\mathrm{SpT}$ & Multiple $^{a}$ \\
\hline 3284 & 2321395090088371456 & $00: 07: 13.73$ & $-28: 54: 38.94$ & 15.22 & 15.82 & 17.21 & 14.67 & 12.22 & 3.60 & M3 & $\mathrm{c}$ \\
\hline 4258 & 2320971606313124352 & 00:06:51.14 & $-29: 02: 58.37$ & 16.00 & 16.57 & 17.92 & 15.43 & 13.02 & 3.55 & M3 & - \\
\hline 5249 & 2321010329738234112 & 00:06:09.05 & $-29: 09: 10.48$ & 8.41 & 7.89 & 7.89 & 7.93 & 8.00 & -0.10 & B9 & - \\
\hline 6610 & 2320945699070402816 & $00: 06: 53.50$ & $-29: 21: 13.25$ & 14.26 & 14.86 & 15.75 & 13.95 & 11.97 & 2.89 & K8 & - \\
\hline 6761 & 2320950333339180544 & $00: 07: 25.36$ & $-29: 22: 25.12$ & 15.38 & 16.05 & 17.33 & 14.82 & 12.43 & 3.62 & M3 & $\mathrm{c}$ \\
\hline
\end{tabular}

$\overline{\mathrm{a}} \mathrm{c}=\mathrm{CMD} ; \mathrm{r}=\mathrm{RV}$. For example, 'cr' would indicate a system that was highlighted as a likely multiple system by both methods.

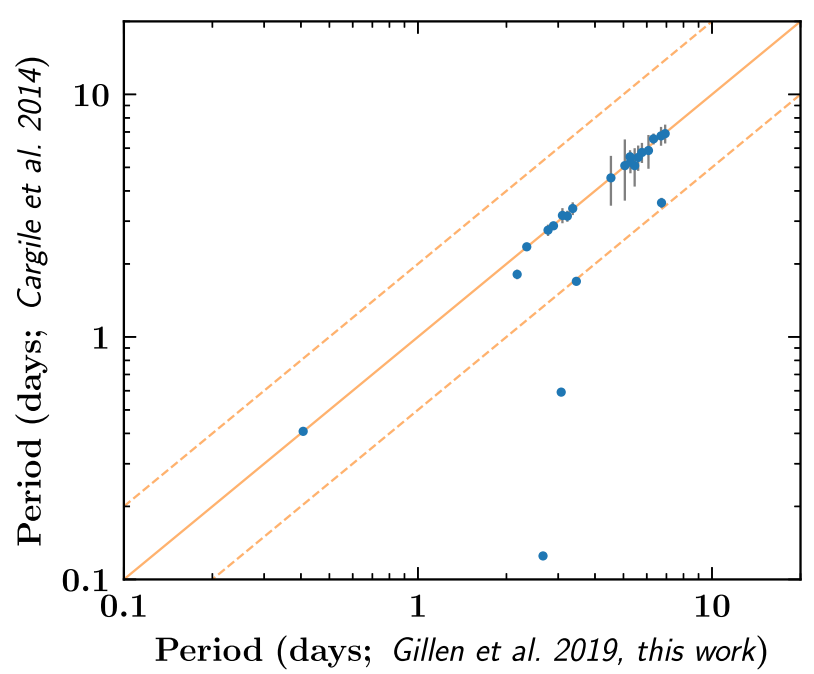

Figure 6. Comparison between our rotation periods and those of $\mathrm{C} 14$ for 23 stars with detected periods in both surveys. Of these, 18 have periods that agree to within 10 percent or better. The solid and two dashed orange lines show the 1:1 period match, and the 2:1 and 1:2 harmonics. The period uncertainties reported in this work are typically the size of the points or smaller.

two stars (NGTS IDs 14186 and $10246^{5}$ ) lie on the 1:2 harmonic, with $\mathrm{C} 14$ reporting periods that are half of ours; one star (NGTS ID $8749^{5}$ ) has a period in C14 that lines up with an alias of our period in the LS periodogram; and the last outlier (NGTS ID $12641^{5}$ ) has a very short period in $\mathrm{C} 14$, which is indicative of a binary system, but we do not identify it as such here and find its rotation period places it on the well-defined single-star sequence for its colour. From comparison of our light curves with those in $\mathrm{C} 14$, and the fact that all three of our methods agree for the stars not lying on the 1:1 relation, we favour our rotation periods for all stars.

\section{IDENTIFYING MULTIPLE STARS}

We identify binary and higher order multiple stars using two complementary approaches: (i) fitting the single-star cluster sequence in colour-magnitude space and identifying stars lying above this trend and (ii) cross-matching with literature RV surveys. We describe each method in Sections 4.1 and 4.2.

\subsection{CMD fitting}

We created four CMDs to help us identify binaries with different mass ratios. These were Gaia $M_{\mathrm{G}}$ versus $\left(G_{\mathrm{BP}}-G_{\mathrm{RP}}\right)_{0}, M_{\mathrm{G}}$ versus
$\left(G-K_{\mathrm{s}}\right)_{0}, 2$ MASS $M_{\mathrm{K}_{\mathrm{s}}}$ versus $\left(G_{\mathrm{BP}}-G_{\mathrm{RP}}\right)_{0}$, and $M_{\mathrm{K}_{\mathrm{s}}}$ versus $(G$ $\left.-K_{\mathrm{s}}\right)_{0}$. From the optical $M_{\mathrm{G}}$ versus $\left(G_{\mathrm{BP}}-G_{\mathrm{RP}}\right)_{0} \mathrm{CMD}$, relatively equal mass binaries are typically easy to identify as their colour remains roughly constant but their magnitude increases, with an equal-mass binary lying $0.75 \mathrm{mag}$ above the single-star sequence. Low mass ratio binaries are harder to detect in such an optical $\mathrm{CMD}$, however. Taking the example of a $\mathrm{G}$ star with an M-dwarf companion, the $\mathrm{M}$ dwarf will not affect the optical $\mathrm{G}$ magnitude much, and will only shift the BP-RP colour slightly redder, as the M dwarf contributes more to the red optical flux than the blue. Such a small colour shift is difficult to detect even with the precision of the Gaia data. An $M_{\mathrm{K}_{\mathrm{s}}}$ versus $\left(G-K_{\mathrm{s}}\right)_{0}$ CMD is better suited for identifying low mass ratio binaries, as the presence of a low-mass companion will more strongly contribute to the infrared $K_{\mathrm{S}}$ band and therefore shift the binary in both $K_{\mathrm{s}}$ magnitude and $G-K_{\mathrm{s}}$ colour. By using four CMDs, we can track the relative positions of each star in the four planes, and gain a better handle on their likelihood of being a multiple-star system.

With the precision of Gaia data, stellar evolution models struggle to fit the exact shape of cluster CMDs at a given age and metallicity (see e.g. B18); this motivated us to use a flexible non-parametric model. For each CMD, we iteratively fit the whole cluster sequence using a GP with a stochastically driven damped SHO kernel, as implemented in CELERITE and EXOPLANET, and fixed the quality factor at $Q=1 / 2$, which approximates the well-known Matern-3/2 kernel (Rasmussen \& Williams 2006). At each step, we perform a running median filter on the residuals of the fit and reject $3 \sigma$ outliers above the GP model in the next iteration, with this process typically converging towards an MAP fit to the single-star cluster sequence within $\sim 5$ iterations.

Fig. 7 shows the $M_{\mathrm{G}}$ versus $\left(G_{\mathrm{BP}}-G_{\mathrm{RP}}\right)_{0}$ and $M_{\mathrm{K}_{\mathrm{s}}}$ versus $\left(G-K_{\mathrm{s}}\right)_{0}$ CMDs (left and right, respectively). While all four CMDs display a clear single-star cluster sequence with multiple-star outliers above the trend, the exact positions of individual multiple systems in each CMD can differ quite significantly due to their component mass ratios. We identify multiple-star systems as those that lie at least $3 \sigma$ above the single-star GP sequence in any one plane. In practice, if a system was an outlier in one plane, it was typically an outlier in two or more planes. From the four CMDs, we identify 39 multiple-star systems from the 170 stars with NGTS light curves.

\subsection{Cross-matching with literature radial velocity surveys}

We cross-matched our cluster sample with literature RV surveys, namely those of Mermilliod et al. (2008, 2009) and González \& Levato (2009), and identify seven SB1 and two SB2 binaries within our members that have NGTS light curves. These systems are 

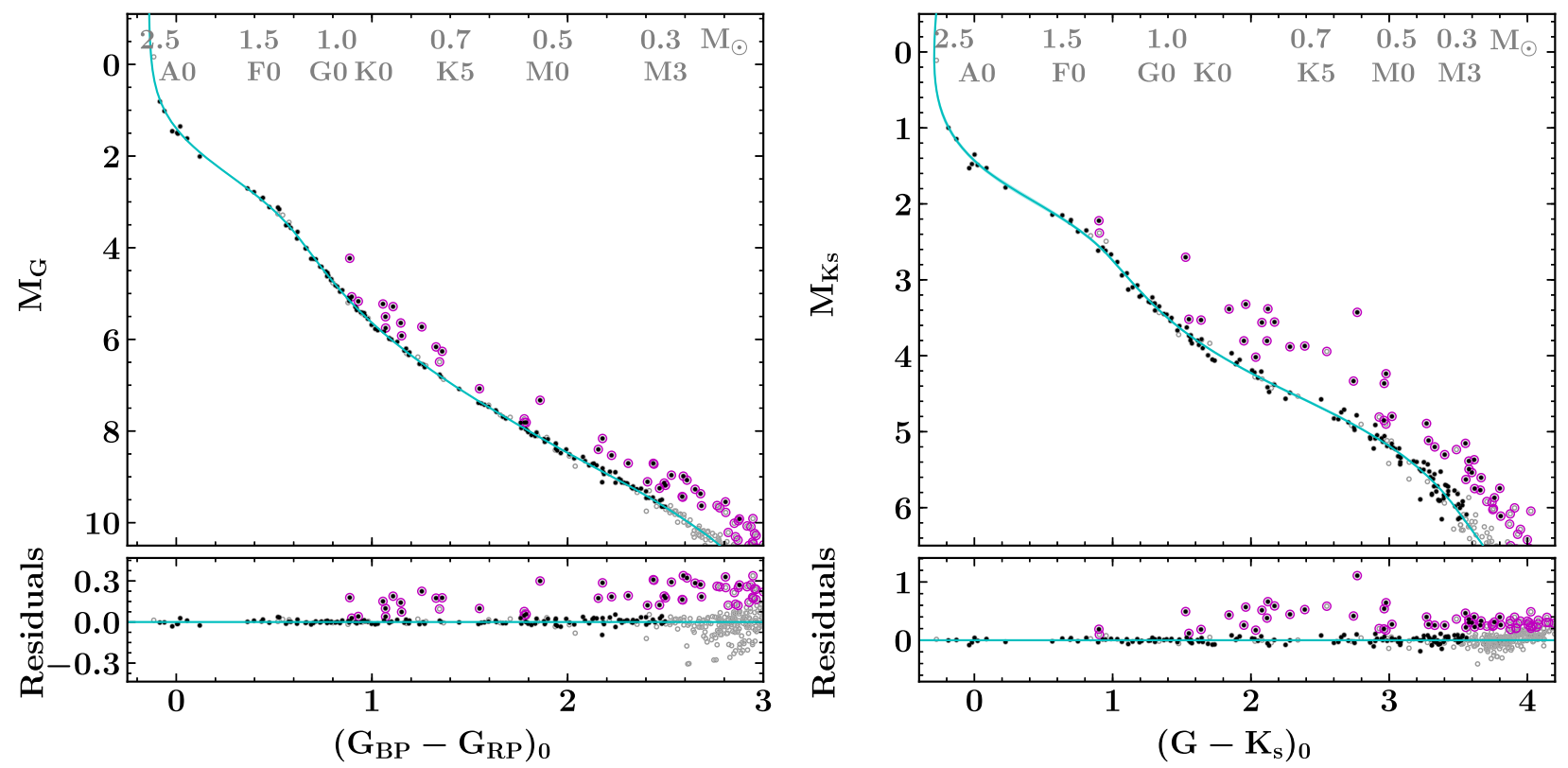

Figure 7. CMDs of Blanco 1. Left: Absolute G versus dereddened $G_{\mathrm{BP}}-G_{\mathrm{RP}} \mathrm{CMD}$ of all Blanco 1 stars (open grey circles) and those with NGTS light curves highlighted (filled black circles). The single-star locus has been estimated by iteratively fitting the cluster sequence using a GP (cyan). Stars that are $3 \sigma$ outliers are circled in magenta and are likely multiple-star systems. Below are the residuals of the fit as a function of colour, where 'residual' means the smallest linear distance from the GP model rather than vertical magnitude displacement above the single-star sequence. Right: Same for absolute $K_{\mathrm{S}}$ versus dereddened $G-K_{\mathrm{S}} \mathrm{CMD}$.

identified in Tables 1 and 2. Three of the five periodic systems are also identified as binaries from our CMD analysis. In the following sections, we use only the photometric multiples identified using our CMD analysis, and therefore do not consider the other two periodic systems (IDs 9992 and 11872) as multiples, although we do list them as such in Table 1. This is for two reasons: (i) the literature RV surveys of Blanco 1 are not complete in terms of either membership or spectral type, which makes the identified multiples hard to interpret statistically, and (ii) in Section 6, we compare to the Pleiades, for which we identify multiples using our CMD analysis only, and therefore use the same multiple-star criteria for Blanco 1 for fair comparison.

\section{ROTATION IN BLANCO 1}

\subsection{Colour-period distribution}

\subsubsection{FGK stars with masses $0.7 M_{\odot} \lesssim \mathrm{M} \lesssim 1.2 M_{\odot}$}

The rotation period distribution of stars in Blanco 1 as a function of $\left(G-K_{\mathrm{s}}\right)_{0}$ colour is shown in Fig. 8. In the left-hand plot, we show all stars for which we determined rotation periods and on the right we remove stars identified as likely multiple systems (see Section 4) to highlight the trend in the (apparently) single-star population. For these single stars, there is a clear mass dependence in the rotation distribution, which is especially evident for the mid-F to mid-K stars $\left(1.0<\left(G-K_{\mathrm{s}}\right)_{0}<2.5,1.2 \mathrm{M}_{\odot} \gtrsim M \gtrsim 0.7 \mathrm{M}_{\odot}\right)$, as they follow a tight rotation sequence between 2 and $10 \mathrm{~d}$. Stars lying on this tight sequence are almost exclusively likely single stars, whereas those lying under the sequence at shorter rotation periods are almost exclusively likely multiple-star systems.

The origin of this dichotomy is not well understood. All of the multiples identified here are photometric multiples, and hence are moderate-to-high mass ratio systems. ${ }^{6}$ In the field, the mass ratio distribution for multiples shows a preference towards high mass ratio pairs and that these occur more frequently in relatively close configurations (Raghavan et al. 2010). One might expect therefore that many of the photometric multiples identified here also have relatively close separations. This may be important because stars that form in close-separation $(\lesssim 100 \mathrm{au})$ multiples are thought to have their circumstellar disc lifetimes truncated due to the presence of their close companions (e.g. Patience et al. 2002; Meibom, Mathieu \& Stassun 2007; Daemgen, Correia \& Petr-Gotzens 2012; Daemgen et al. 2013). Reduced disc lifetimes imply shorter phases of magnetic disc braking, and hence an earlier spin-up towards the ZAMS that presumably results in faster (and perhaps more widespread) rotational velocities at the start of MS evolution compared to single stars of the same mass. Alternatively, the star formation process may deposit angular momentum differently in close multiple systems compared to single or wide multiples (Larson 2003), which may also result in different rotation period distributions on the ZAMS. In any case, surface rotational velocities decrease as stars evolve off the ZAMS due to angular momentum loss through magnetized stellar winds and redistribution within the stellar interior. If stars in close multiples do possess a different rotation period distribution compared to single stars on the ZAMS, it follows that this will persist during the early MS before all nontidally locked stars eventually converge towards the same rotation period distribution. It remains unclear, on both observational and theoretical grounds, how long such convergence might take, but it does not appear to have occurred by the age of Blanco 1 ( $\sim 115 \mathrm{Myr})$,

\footnotetext{
${ }^{6}$ We note that one of the two RV-detected binary systems, which was not also a photometric multiple, sits on the well-defined sequence of apparently single stars, while the other sits above (possibly because the secondary component is responsible for the modulation signal).
} 

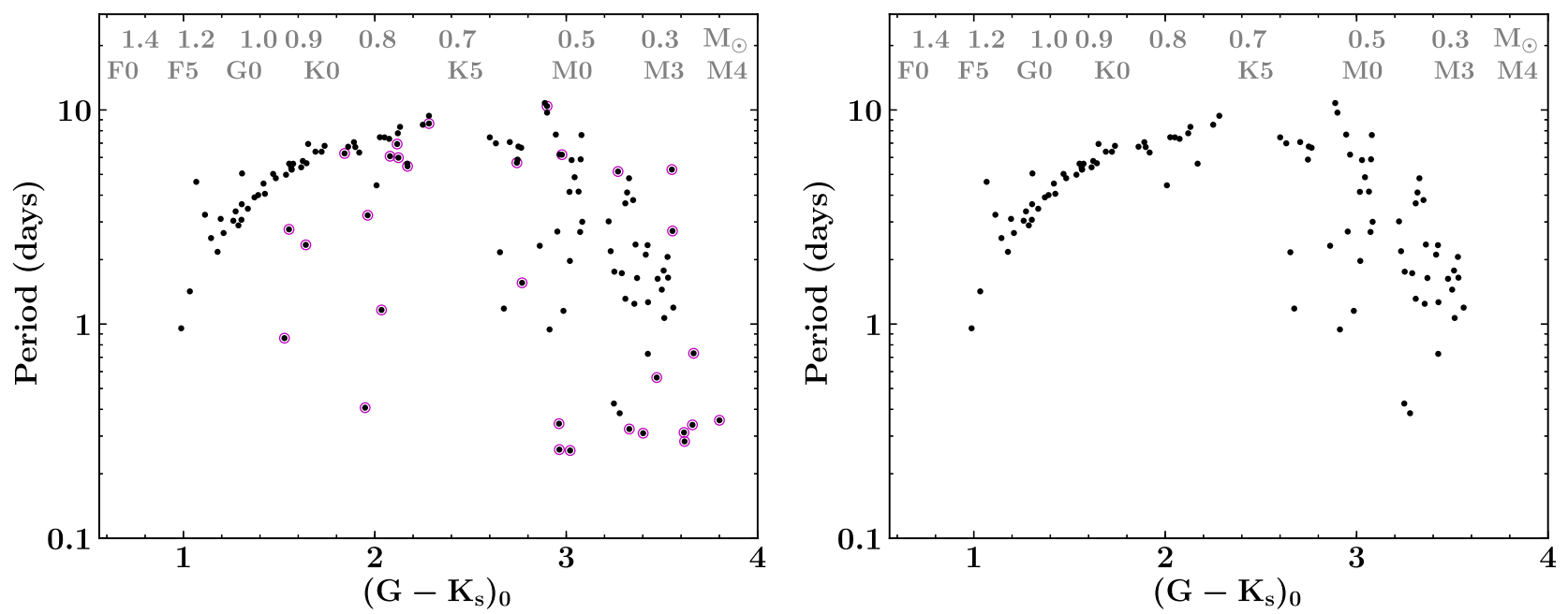

Figure 8. Rotation period versus dereddened $G-K_{\mathrm{S}}$ colour for stars in Blanco 1. Left: All stars with detected rotation periods (black points), with our identified multiple stars circled in magenta. Stellar mass $\left(\mathrm{M}_{\odot}\right)$ and spectral type are indicated at the top. Right: Only the apparently single stars to highlight the clear rotation sequence between $1.1<\left(G-K_{\mathrm{s}}\right)_{0}<2.3\left(1.2 \mathrm{M}_{\odot} \gtrsim M \gtrsim 0.75 \mathrm{M}_{\odot}\right)$. Mass-dependent angular momentum evolution is strongly imprinted in the Blanco 1 sample.

at least for $\mathrm{G}$ and $\mathrm{K}$ stars. By the age of Praesepe (700-800 Myr), however, it seems as though essentially all non-tidally locked FGK stars, irrespective of their hierarchy, have converged to a welldefined rotation sequence (Rebull et al. 2017). Further observations of clusters, specifically those with ages between $\sim 100$ and $800 \mathrm{Myr}$, are needed to constrain this convergence time-scale. It is worth noting that some of the Blanco 1 multiples identified here may be very close-separation binaries (orbital periods less than $\sim 10-15 \mathrm{~d}$ ), whose rotational evolution will be driven by tidal forces that act to synchronize the stellar rotation periods to that of the binary orbital period, and hence will never converge on to the single-star rotation sequence. Based on very limited RV monitoring by Mermilliod et al. (2009) of five of these multiples, however, this does not appear to be the case for all systems: while two are spectroscopic binaries, three appear to have essentially flat RVs to within their uncertainties. Further RVs are needed to confirm this tentative statement.

We highlight two stars (with $\left(G-K_{\mathrm{s}}\right)_{0}$ colours of 2.0 and 2.3) that lie below the single-star trend, but do not appear to be photometric or spectroscopic multiples. We suggest that both of these systems are either (i) multiples containing very low mass companions, such that they are not identified by us as multiples, or (ii) single stars whose angular momentum loss has been inhibited at some point during their evolution. We consider the first option as the more likely given the correlation between multiplicity and faster rotation in this mass range.

Finally, we note that roughly half of $\sim \mathrm{F} 6-\mathrm{K} 3$ stars are expected to reside in binary or higher order systems (Raghavan et al. 2010). We identify $\sim 20$ per cent of the FGK stars as moderate-to-high mass ratio multiples, which suggests that some of the apparently single stars in our sample that lie on the well-defined rotation sequence are likely low mass ratio multiples. Given this, we surmise that the presence of a low-mass companion alone is not sufficient to significantly affect the angular momentum evolution of the primary star. This is evidenced by one of the two RV-detected multiples that was not also a photometric multiple, which lies on the welldefined rotation sequence for FGK stars (although we note that the other such system sits above this sequence, possibly because the secondary component is responsible for the modulation signal).

\subsubsection{Late- $K$ and $M$ stars with masses $0.3 M_{\odot} \lesssim \mathrm{M} \lesssim 0.6 M_{\odot}$}

For late-K and $\mathrm{M}$ stars $\left(2.5 \lesssim\left(G-K_{\mathrm{s}}\right)_{0} \lesssim 3.6,0.7 \mathrm{M}_{\odot} \gtrsim M\right.$ $\gtrsim 0.3 \mathrm{M}_{\odot}$ ), there is no well-defined rotation sequence, with the apparently single stars possessing rotation periods ranging from $P$ $<1 \mathrm{~d}$ up to $P \sim 11 \mathrm{~d}$. Within this colour range, multiple-star systems are spread throughout the single-star population, although there is an accumulation of multiples at short $(P<0.5 \mathrm{~d})$ periods. It is likely that this accumulation is, at least partially, a selection effect, as the faintest stars in our sample are mostly multiple systems that are overly bright compared to single stars of the same mass.

\subsection{Light-curve morphology}

\subsubsection{Evolving versus stable modulation patterns}

The NGTS light curves offer an ideal window on to the evolution of young star $(\sim 115 \mathrm{Myr})$ modulation patterns over a well-sampled 200-d baseline. We classified the NGTS light curves based on two metrics: (i) the spread in the dispersion in the phase-folded light curve and (ii) the self-similarity of the modulation pattern throughout the light curve. For the latter metric, we used running windows of three to five rotation periods, folded the data within each window on the global rotation period, and compared the difference in the flux measurements at similar phases throughout the light curve. Both metrics are probing the self-similarity of the data throughout the NGTS light curves, but we found that they were more sensitive to different aspects of the evolution. Combining these two metrics, by adding them in quadrature, provided a reasonably good indication of the level of evolution within a given light curve. We show the results in Fig. 9, where we see a strong mass dependence in the light-curve morphologies: the majority of mid-F to mid-K stars show moderate-to-significant evolution (i.e. varying amplitudes and phase shapes), whereas $\mathrm{M}$ star modulation patterns appear generally stable over $200 \mathrm{~d}$, with a transition between these occurring at late-K spectral types. In Fig. 10, we show three example light curves that highlight the range in evolution present within the Blanco 1 stars, from more to less evolution (top to bottom). 


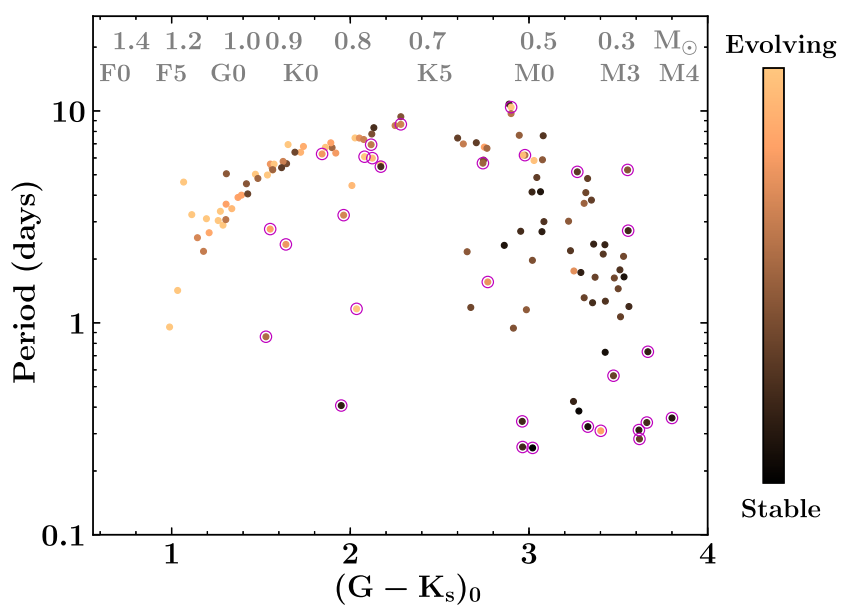

Figure 9. Rotation period versus dereddened $G-K_{\mathrm{S}}$ colour for stars in Blanco 1 coloured by the level of evolution in their light-curve modulation patterns. There is a clear mass dependence to the light-curve morphology evolution, with mid-F to mid-K stars displaying predominantly evolving modulation patterns and $\mathrm{M}$ stars showing typically stable modulation over the 200-d NGTS light curves.

Physically, this difference may be due to a change in the dominant magnetic field morphology between mid-F to mid-K stars and $\mathrm{M}$ dwarfs at this age. It is interesting to note that the change from predominantly evolving modulation patterns to predominantly stable patterns occurs around the same mass as the change from a welldefined rotation sequence to a broad rotation period distribution (i.e. $M \sim 0.6 \mathrm{M}_{\odot},\left(G-K_{\mathrm{s}}\right)_{0} \sim 2.8$, late-K spectral type). This hints at a possible relation between the dominant magnetic field topology and the convergence of stars on to a well-defined rotation sequence at a given age. It would be interesting to investigate this in more detail by comparing the modulation patterns and rotation distributions of clusters spanning a range of ages over the first billion years.

Our ability to detect evolution in the modulation patterns decreases with signal-to-noise ratio $(\mathrm{S} / \mathrm{N})$ and is therefore harder for the fainter M stars. Specifically, it is harder to detect small levels of evolution as the relative noise level is higher for a given modulation amplitude. Significant evolution, which corresponds to the orange colours in Fig. 9, should be detectable for even the faintest stars in our sample. However, as we find that most $M$ stars display stable modulation patterns, it is worth considering whether this finding is robust or is affected by our reduced ability to identify small evolution changes in low-S/N light curves. To show this unambiguously would require injection-recovery tests in simulated NGTS light curves, which is beyond the scope of this work. We note, however, that similar conclusions have been postulated for $\mathrm{M}$ stars in the Pleiades (Stauffer et al. 2016), based on $K 2$ data with higher S/N for M0-M3 spectral types. We therefore suggest that the main conclusions on $\mathrm{M}$ stars here, i.e. that they often display stable modulation patterns over 200 -d periods, are valid.

Finally, we note that $K 2$ campaigns lasted $75 \mathrm{~d}$ and most TESS (Transiting Exoplanet Survey Satellite; Ricker et al. 2014) fields will have $27-d$ coverage. ${ }^{7}$ Within a given $75-d K 2$ window, and even more so within a 27-d TESS window, it would be difficult to see evolution in the modulation shape to the extent that is evident in the NGTS data. Such a long temporal baseline, combined with

\footnotetext{
${ }^{7}$ With longer coverage towards the ecliptic poles where individual sectors
} overlap. this level of sampling and photometric precision, is unprecedented for young stars.

\subsubsection{Spot evolution and/or differential rotation?}

We noticed that for stars displaying significant evolution in their light curves, the strongest LS periodogram peak was often split into two close peaks or had a complex shape. Such split/complex LS peaks have been noted for similarly aged young stars in the Pleiades based on analysis of $K 2$ light curves (Rebull et al. 2016b). This might tentatively suggest that two or more close periodic signals exist in the data, which could be a sign of differential rotation (under the assumption that spot modulation is sinusoidal and spot groups survive throughout most of our light curves) and/or evolution in the spot distributions. The GP MCMC posterior period distributions for such stars are well defined and single peaked, which would support the spot evolution scenario as the simplest explanation. However, we note that the fractional period uncertainties for these variable stars are typically larger than those for stars displaying stable modulation patterns with similar periods and spectral types. ${ }^{8}$ This is probably because the rotation periods of stars with variable modulation patterns are simply less well constrained (given the more complex nature of the variability and hence required flexibility of the GP model), but it could also be because the period distributions are actually the summation of two or more closely overlapping, well-defined period distributions, as one might expect if differential rotation is present and significant enough to increase the spread in the measured rotation period distribution. Given that stars displaying evolving modulation patterns and broader rotation period distributions are typically FGK stars, this would require differential rotation to be more prominent in these stars than in $\mathrm{M}$ dwarfs. Indeed, measured rates of differential rotation have been observed to decrease with stellar temperature (e.g. Barnes et al. 2005; Collier Cameron 2007), with measurements for M dwarfs at or below the fully convective boundary significantly lower than could be detected in the NGTS data of Blanco 1 (e.g. Morin et al. 2008a, b; Reinhold, Reiners \& Basri 2013; Davenport, Hebb \& Hawley 2015). While we feel that the rotation periods of stars with evolving modulation patterns will simply be less well constrained due to their more complex nature, it is certainly plausible that differential rotation is also present and stronger in the FGK stars.

Accurately decoupling spot evolution from differential rotation is troublesome (see e.g. Aigrain et al. 2015, for a discussion based on Kepler light curves of solar-type stars). Differential rotation has, however, been reported in young field stars observed by the Kepler prime mission (Frasca et al. 2011; Fröhlich et al. 2012), based on multispot models that allow the shape of spots to evolve in time, but assume the spots survive throughout the observations. The authors are not aware of modelling efforts that have been applied to young solar-type and low-mass stars that allow evolution in both the shape and existence of different active regions, such that a differential measure of the stellar rotation period is possible from each active region's modulation period during their lifetimes. Unfortunately, the precision and duration of the NGTS data, combined with our limited knowledge of spot lifetimes and behaviour at $\sim 100 \mathrm{Myr}$, do not allow us to distinguish between spot evolution and differential rotation with the current analysis.

\footnotetext{
${ }^{8}$ We note, however, that given the small number of FGK stars displaying
} stable patterns, this assertion is based on low numbers. 

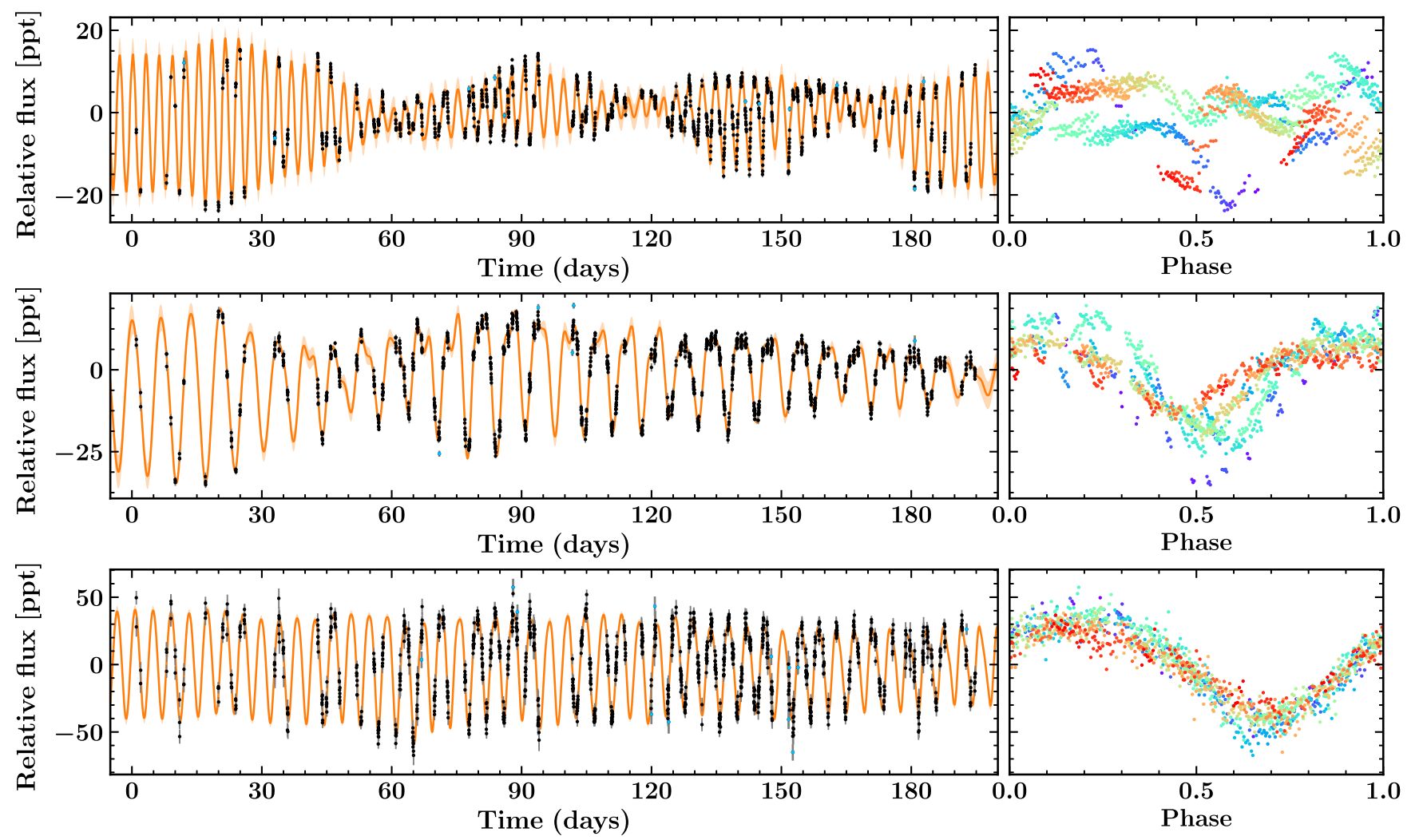

Figure 10. Three example light curves displaying different levels of evolution. Top to bottom: Light curves showing strong evolution, moderate evolution, and stable modulation. In each case, the left-hand panel shows the full light curve with the GP model fit, and the right-hand panel the phase-folded light curve. The rainbow colour scheme represents individual modulation periods from the beginning (indigo) to the end (red) of the light curve.

\subsection{Colour-period-amplitude relation}

Fig. 11 shows the colour-period relation for stars in Blanco 1 coloured by the amplitude of their modulation patterns. Focusing on the single stars, as we move from mid-F to mid-K spectral types there is a slight increase in the average amplitude, which probably reflects the increasing size of the convective outer layer in these stars (see Section 6.4 for further discussion). For the single late-K and early $\mathrm{M}$ stars $\left(2.5 \lesssim\left(G-K_{\mathrm{s}}\right)_{0} \lesssim 3.2\right)$, the faster rotators, which sit below the upper cluster envelope, display higher modulation amplitudes than their more slowly rotating counterparts. This is not exclusively the case, however, as some faster rotators display modest modulation amplitudes. If the inverse correlation between rotation period and modulation amplitude for single stars at a given colour (i.e. mass) is correct, these low-amplitude fast rotators may be either (i) stars that were observed at comparatively low inclination angles or (ii) binary systems that have lower mass ratios than we could detect with our CMD analysis. We note that the binaries we did identify do not show an obvious trend between rotation period and amplitude at a given $\left(G-K_{\mathrm{s}}\right)_{0}$ colour. This is unsurprising as each system will have different modulation signals from the component stars, which may differ in amplitude (depending on the light ratio between the components), and constructively and/or destructively interfere over the course of the NGTS observations (if the components are not tidally locked with negligible differential rotation).

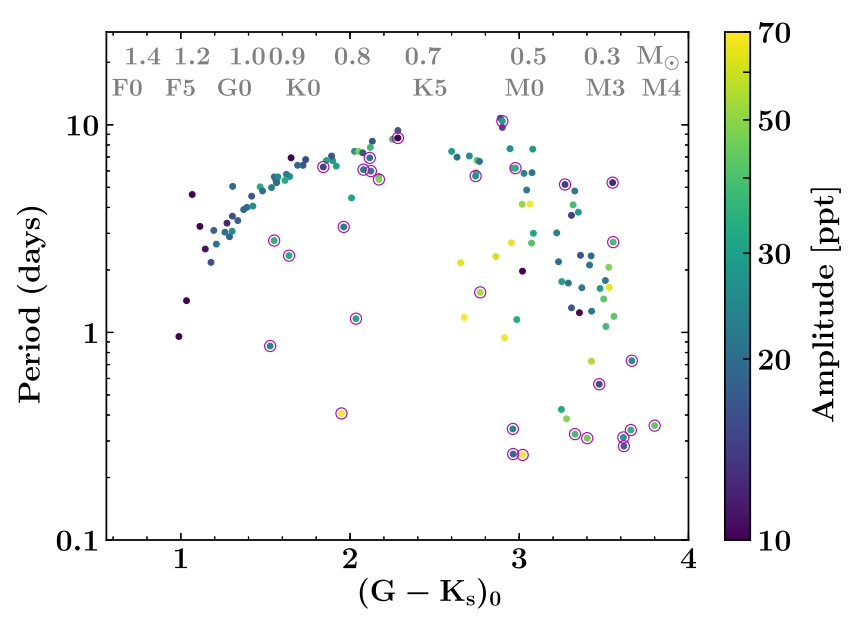

Figure 11. Rotation period versus dereddened $G-K_{\mathrm{S}}$ colour for stars in Blanco 1 coloured by the amplitude of their light-curve modulation patterns. Most notably for single late-K and early M stars $(2.5 \lesssim(G-$ $\left.K_{\mathrm{S}}\right)_{0} \lesssim 3.2$ ), there appears to be a correlation between rotation period and modulation amplitude, with the faster rotators, which sit below the upper cluster envelope, displaying higher modulation amplitudes than their more slowly rotating counterparts. Photometric multiple stars are circled in magenta. 

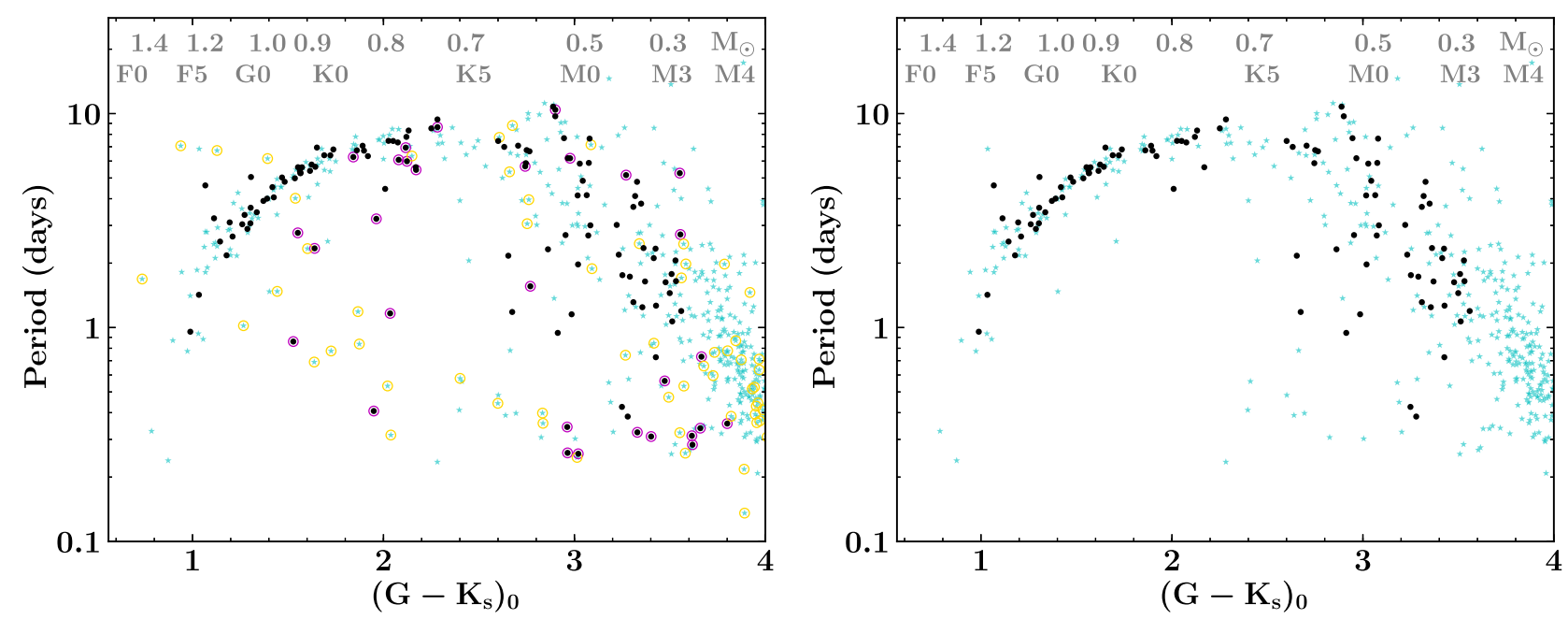

Figure 12. Rotation period versus dereddened $G-K_{\mathrm{s}}$ colour for stars in Blanco 1 and the Pleiades. This is the same as Fig. 8 with the addition of the Pleiades data, which have been dereddened assuming $E(B-V)=0.045$ for the cluster (B18). Left: All stars with detected rotation periods (black points for Blanco 1 and cyan stars for the Pleiades), with our identified multiple-star systems highlighted (magenta circles for Blanco 1 and gold circles for the Pleiades). Right: Only the apparently single stars to highlight the clear rotation sequences between $1.1<\left(G-K_{\mathrm{s}}\right)_{0}<2.3\left(1.2 \mathrm{M}_{\odot} \gtrsim M \gtrsim 0.75 \mathrm{M}_{\odot}\right)$. Mass-dependent angular momentum evolution is strongly imprinted in both clusters.

We refrain from postulating about the lowest mass stars $((G$ $\left.-K_{\mathrm{s}}\right)_{0} \gtrsim 3.2$ ) because we become increasingly more sensitive to higher amplitude variables for the faintest stars.

\section{COMPARING BLANCO 1 AND THE PLEIADES}

\subsection{Colour-period distribution}

Stellar rotation in the Pleiades has been extensively studied (e.g. Hartman et al. 2010; Covey et al. 2016b; Rebull et al. 2016a, b; Stauffer et al. 2016) and now Blanco 1 also possesses a wellconstrained distribution of rotation periods. Given the very similar age estimates for these clusters, we compare their rotation period distributions to understand the level to which mass-dependent angular momentum loss mechanisms are imprinted on stars at 〜10-115 Myr.

We cross-matched the Gaia DR2 Pleiades membership list with stars from Rebull et al. (2016a), which resulted in 589 Pleiads with measured rotation periods. ${ }^{9}$ We also performed our CMD fitting technique on the Pleiades to identify likely multiple systems. The colour-period distributions of both clusters are shown in Fig. 12 (full distributions in the left-hand panel and only the apparently single stars in the right-hand panel).

The period distributions in both clusters are strikingly similar. Fig. 12 (right-hand plot) shows that essentially all single stars in the mid-F to mid-K spectral range $\left(0.7 \mathrm{M}_{\odot} \lesssim M \lesssim 1.2 \mathrm{M}_{\odot}\right)$ lie on tight cluster sequences. This rotation sequence is slightly tighter for Blanco 1 than for the Pleiades, which we attribute to a combination of a longer observation baseline ( $200 \mathrm{~d}$ versus $75 \mathrm{~d}$ ) and the novel period estimation methods applied here.

\footnotetext{
${ }^{9}$ The Rebull et al. (2016a) sample contained 759 likely members. While some of the stars not in the Gaia DR2 membership list may be non-members, many are likely Pleiads that reside in multiple-star systems, which may be affecting the DR2 astrometric solution. It would be interesting to revisit these stars in future data releases.
}

\subsubsection{FGK stars with intermediate rotation periods}

In addition to the tight cluster sequence, previous Pleiades rotation studies identified two populations of stars below this sequence, termed intermediate and fast rotators based on the magnitude of their displacement below the main cluster sequence (see e.g. fig. 2 in Stauffer et al. 2016). Following our Gaia DR2-based membership selection from B18, we do not see the intermediate sequence as strongly, which suggests that either these were mainly non-members or their Gaia DR2 astrometric solutions were suspect, which could be due to the effect of binarity, as Stauffer et al. (2016) noted these stars were preferentially displaced above the CMD cluster sequence.

There are a few stars that remain in this intermediate rotator sequence, however, which can be seen sitting just below the welldefined single-star sequence (two in Blanco 1 and two in the Pleiades). We suspect these are likely binaries with low-mass companions and therefore were not flagged by our photometric identification methods. It is interesting to note that these stars appear to follow a trend with rotation periods $\sim 2-3 \mathrm{~d}$ shorter than the main rotation sequence, so intermediate 'sequence' is perhaps a relevant term. However, with only four such stars, we refrain from making further statements; additional clusters with similar ages are needed to shed further light on this potential small subpopulation.

\subsubsection{The apparent kink in the Pleiades single-star sequence around a spectral type of $\sim K 5$}

The Pleiades data in Fig. 12 show an apparent kink in the single-star sequence around a spectral type of $\mathrm{K} 5\left(\left(G-K_{\mathrm{s}}\right)_{0} \sim 2.5\right)$, with the upper envelope of the late-K stars reaching longer rotation periods than earlier spectral types. This kink was first noted by Stauffer et al. (2016). Here, we see the kink even more clearly following the Gaia DR2 membership selection, suggesting that it is probably a real phenomenon within the cluster sample (assuming the rotation periods for these stars are accurate). There is a dearth of late-K stars in Blanco 1, which means we cannot strongly comment on the presence of such a kink in this cluster, although we note that the Blanco 1 rotation period distribution within this mid-to-late 


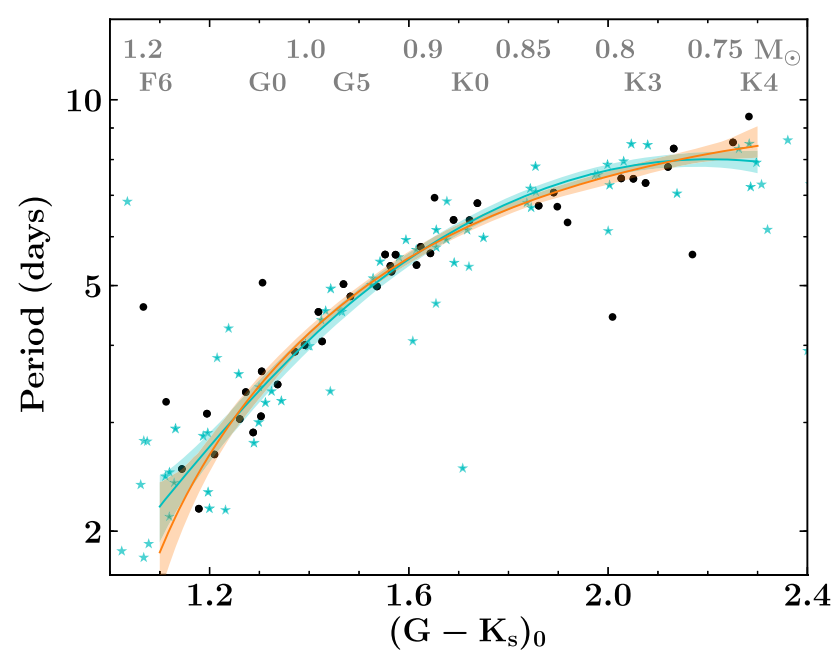

Figure 13. GP fits to the single-star rotation sequences in Blanco 1 (black points, orange model) and the Pleiades (cyan stars, cyan model). The lines and shaded regions indicate the mean and $2 \sigma$ confidence interval of the posterior GP distribution. The rotation sequences of Blanco 1 and the Pleiades are consistent to within their uncertainties across the mid-F to mid-K spectral range $\left(1.2 \mathrm{M}_{\odot} \gtrsim M \gtrsim 0.75 \mathrm{M}_{\odot}\right)$.

$\mathrm{K}$ spectral range $\left(2.3 \lesssim\left(G-K_{\mathrm{S}}\right)_{0} \lesssim 2.9\right)$ is consistent with the Pleiades, and hence also consistent with the presence of such a kink.

\subsection{Fitting the single FGK star cluster sequences}

We fit the rotation periods of single mid-F to mid-K stars in Blanco 1 and the Pleiades to assess the similarity between the rotation sequences in these two clusters. For this, we focus on stars between $1.1<\left(G-K_{\mathrm{s}}\right)_{0}<2.3$, as these ranges encompass the most well defined section of the rotation sequences, and are well populated by stars in both clusters.

Fig. 13 shows our fits to both cluster sequences. We opted to use a GP model because this marginalizes over an ensemble of functions, which is more general than using gyrochronology or polynomial models, but also includes (models similar to) them. We use a squared exponential kernel, as implemented in GEORGE (Ambikasaran et al. 2015), and perform a two-stage fit, first running an iterative MAP fit with $3 \sigma$ outlier rejection, before running an MCMC fit to each cluster sequence (5000 steps, 200 walkers, with a 3000-step burn-in) using EMCEE (Foreman-Mackey et al. 2013). Iterative outlier rejection was performed, even though we fit only the (apparently) single-star sequences, because our multiple-star identification methods are not sensitive to all multiple-star mass ratios and separations. It is possible therefore that some of the fitted stars reside in multiple-star systems where their angular momentum evolution has been affected by hitherto unidentified companions, and hence should not be included in this analysis, which is seeking to focus on the single-star rotation sequences in both clusters.

It is clear that the Blanco 1 and Pleiades rotation sequence fits (orange and cyan lines and shaded regions) are consistent with each other. ${ }^{10}$ It follows that the angular momentum evolution of mid-

\footnotetext{
${ }^{10}$ Performing a similar fit with quadratic polynomial models also suggests the two rotation sequences are consistent, although a less extensive range of models is explored.
}

F to mid-K stars follows a well-defined pathway that is strongly imprinted by $\sim 100 \mathrm{Myr}$, irrespective of their individual angular momentum evolution histories. Furthermore, CMD isochronal age estimates for both clusters are essentially identical (to within $\sim 5 \mathrm{Myr}$ in B18). From their rotation sequence agreement, it follows that their gyrochronological ages would also agree, which is quite encouraging for gyrochronology relations seeking to identify a singular rotation-mass-age relationship that has thus far proved elusive (Angus et al. 2015). We note, however, that the scatter in the two sequences is larger than the posterior confidence intervals, which suggests that there is some intrinsic scatter in the data above a singular period-colour relation. Performing a detailed gyrochronological modelling of the Blanco 1 and Pleiades singlestar sequences is beyond the scope of this paper; we leave this to future work.

\subsection{Assessing the similarity between the low-mass populations}

While it is intuitively straightforward to show that the mid-F to mid-K stars in Blanco 1 and the Pleiades follow a consistent trend (to within the precision of the current rotation period data), it is harder to show this for the lower mass populations. This is primarily because (i) the low-mass stars do not follow a welldefined sequence; (ii) multiple-star contamination will likely be higher because our identification methods are less sensitive for $\mathbf{M}$ stars, as they possess an intrinsically broader spread in luminosities; and (iii) the NGTS observations of Blanco 1 do not probe as deep as the $K 2$ Pleiades data, which means we are progressively more sensitive to larger amplitude variables in Blanco 1 compared to the Pleiades for the latest spectral types. Given these complicating factors, we opt to carry out a simpler test of similarity between the two populations.

We perform two-sample Kolmogorov-Smirnov (KS) and Anderson-Darling (AD) tests (e.g. Feigelson \& Babu 2012) on the period distributions of the low-mass single stars $(2.6<(G$ $\left.\left.-K_{\mathrm{s}}\right)_{0} \leq 3.6\right)$. Both $\mathrm{KS}$ and $\mathrm{AD}$ tests are non-parametric and distribution free. The main difference, in the context of this work, is that $\mathrm{AD}$ is more sensitive to differences near the edges of distributions.

We note that neither KS nor AD tests are strictly valid when data are distributed in two (or more) dimensions, as is the case here, because there is no unique way to order the data. This means that two data sets can yield the same empirical distribution function while possessing different distributions, thereby invalidating the KS or $\mathrm{AD}$ test statistics. To account for this, we perform $\mathrm{KS}$ and $\mathrm{AD}$ tests on data within small $\Delta\left(G-K_{\mathrm{s}}\right)_{0}=0.2$ colour slices, on the basis that any trend within such a small colour range is negligible given the spread and sampling of the data. Under this assumption, we find that both the KS and AD tests cannot reject the null hypothesis that the Blanco 1 and Pleiades low-mass rotation period distributions are drawn from the same parent population. The AD test is generally less confident than the KS test, given that it is more sensitive to stars with short and long periods, and there appears to be a dearth of fast ( $\sim 0.3-0.6 \mathrm{~d})$ single-star rotators in Blanco 1 compared to the Pleiades in this mass range.

\subsection{Probing star-spot distributions across FGKM stars}

Fig. 14 shows the measured amplitudes of the modulation patterns as a function of $\left(G-K_{\mathrm{s}}\right)_{0}$ colour for both Blanco 1 and the Pleiades. Amplitude is defined here as the 10th to 90th flux percentile of the 


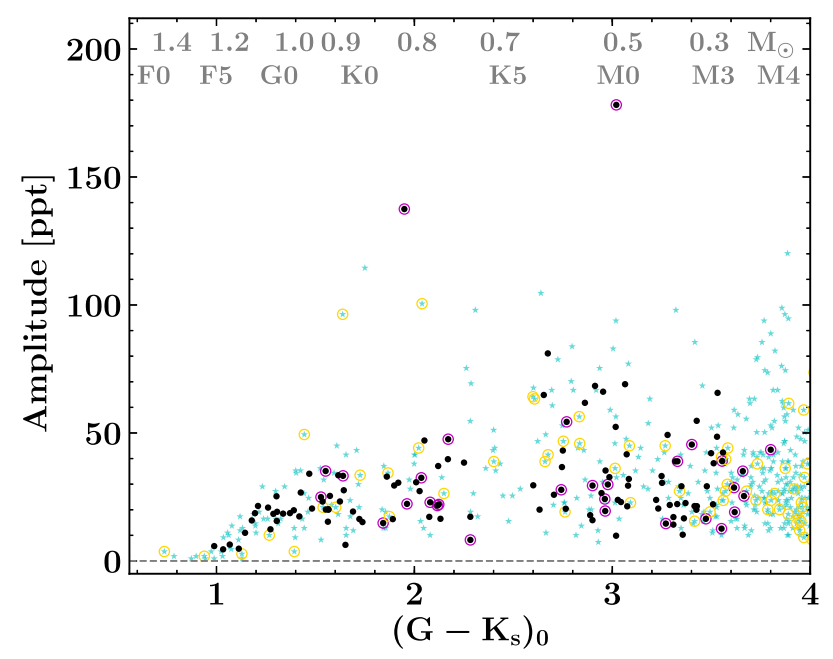

Figure 14. Colour-amplitude relation for Blanco 1 and the Pleiades. Blanco 1 stars are represented by black points, with multiple stars circled in magenta, and Pleiads are indicated by cyan stars, with multiples circled in gold. There is no clear distinction between the modulation amplitudes of single and multiple stars, except that very large amplitude variables are preferentially found in multiple systems. Stellar masses $\left(\mathbf{M}_{\odot}\right)$ and spectral types are indicated at the top. At a spectral type of $\sim F 5$, variability amplitudes start to increase, which we attribute to the emergence of sufficiently deep convective envelopes that can drive and sustain a significant magnetic dynamo, and hence give rise to the star-spot distributions whose longitudinal inhomogeneity drives the observed modulation patterns.

GP model (Blanco 1) and the data (Pleiades). ${ }^{11}$ Moving from left to right on the plot, the amplitude of photometric variability shows a clear increase around a spectral type of F5 $\left(\left(G-K_{\mathrm{s}}\right)_{0} \sim 1.0\right)$. We attribute this to the emergence of sufficiently deep convective envelopes (Wilson 1966), which drive the magnetic dynamos that in turn give rise to surface star-spot distributions (whose longitudinal inhomogeneity drives the observed rotational modulation patterns). This increase in modulation amplitude around F5 spectral type was also noted in the Pleiades (Rebull et al. 2016a). Once convective envelopes become sufficiently deep $\left(\left(G-K_{\mathrm{s}}\right)_{0} \sim 1.4\right.$, early $\mathrm{G}$ spectral type), there is no clear trend between modulation amplitude and stellar mass. The amplitudes of mid-G to mid-M stars are predominantly spread between 10 and $50 \mathrm{ppt}$ with a scattering of higher amplitude variables. However, as shown in Fig. 11, late$\mathrm{K}$ and early $\mathrm{M}$ stars $\left(2.5 \lesssim\left(G-K_{\mathrm{s}}\right)_{0} \lesssim 3.2\right)$ in Blanco 1 show a tentative trend where faster rotators, which sit below the upper cluster envelope, typically display higher modulation amplitudes than their more slowly rotating counterparts. This is the primary driver for the scattering of higher amplitude variables in this colour range in Fig. 14.

Finally, we note that the variability observed in the four earliest spectral type stars in Blanco $1\left(\left(G-K_{\mathrm{s}}\right)_{0} \lesssim 1.1\right)$ could result from pulsations rather than rotational modulation. ZAMS $\mathrm{F}$ stars have temperatures that allow a range of pulsational variability (e.g. $\delta$ Scuti and $\gamma$ Doradus), with $\gamma$ Dor stars possessing pulsation periods (0.4-3.0 d) that overlap with the single-star rotation sequence at this mass (Kaye et al. 1999; Balona et al. 2011; Stauffer et al. 2016).

\footnotetext{
${ }^{11}$ We opted to use our GP model prediction rather than the Blanco 1 data themselves because the NGTS data are not continuous whereas the GP model is, which makes it a more appropriate comparison to the continuous space-based Pleiades data.
}

Given their low variability amplitudes, we cannot easily distinguish between the two variability mechanisms for these stars.

\section{CONCLUSIONS}

We conducted a $\sim 200$-d photometric monitoring campaign of the $\sim 115$-Myr-old Blanco 1 open cluster with NGTS. We determined rotation periods for 127 stars spanning F5-M3 spectral types $\left(0.3 \mathrm{M}_{\odot} \lesssim M \lesssim 1.2 \mathrm{M}_{\odot}\right)$, which increases the number of rotation periods in the cluster by a factor of four.

We used three independent methods to estimate rotation periods: GP regression, G-ACF, and LS periodogram. We find that the GP and G-ACF methods are better suited to estimating rotation periods for solar-type stars that display evolving modulation patterns, as these methods are more flexible than LS. All three methods perform well for stars with stable modulation patterns. In addition to estimating rotation periods, we identified binary and higher order multiple-star systems by fitting the cluster sequence in colourmagnitude space and cross-matching with literature RV surveys.

The rotation period distribution of F5-M3 stars in Blanco 1 shows three main features: (i) single stars between mid-F and mid$\mathrm{K}$ follow a well-defined rotation sequence from $\sim 2$ to $10 \mathrm{~d}$; (ii) the photometric multiples within this spectral type range typically sit below the single-star sequence with shorter rotation periods; and (iii) the late- $\mathrm{K}$ and $\mathrm{M}$ stars possess a broader spread of rotation periods between $\sim 0.3$ and $10 \mathrm{~d}$ with multiple stars spread throughout this distribution.

The fact that mid-F to mid-K photometric multiples have faster rotation rates than their single-star counterparts may suggest that the presence of a close companion with a moderate-to-high mass ratio inhibits angular momentum loss mechanisms during the early PMS, and this signature has not been erased at $~ 100$ Myr.

We find that the majority of mid-F to mid-K stars display modulation patterns that show moderate-to-significant evolution in amplitude and/or phase shape. In contrast, most M0-M3 stars appear to possess reasonably stable modulation signals. This difference could arise from different dominant magnetic field morphologies in mid-F to mid-K stars compared to $\mathrm{M}$ dwarfs at this age, with the transition occurring at late-K spectral types. Interestingly, this morphological change coincides with the shift from a well-defined rotation sequence (mid-F to mid-K stars) to a broad rotation period distribution (M stars) at this age. This hints at a possible relation between magnetic field topology and convergence on to a welldefined rotation sequence at a given age.

Finally, we compared our rotation period distribution for Blanco 1 to the similarly aged Pleiades. We find that the single-star populations in both clusters possess consistent rotation period distributions, which suggests that the angular momentum evolution of stars follows a well-defined pathway that is, at least for mid-F to mid-K stars, strongly imprinted by $\sim 100 \mathrm{Myr}$. This is quite encouraging for gyrochronology relations seeking to identify a singular rotation-mass-age relationship.

\section{ACKNOWLEDGEMENTS}

EG thanks Cristina Blanco for insight that helped shape the direction of this paper, and Richard Booth, Mihkel Kama, Laetitia Delrez, Suzanne Aigrain, John Stauffer, and Luisa Rebull for enjoyable and informative discussions. This research is based on data collected under the NGTS project at the ESO La Silla Paranal Observatory. The NGTS facility is funded by a consortium of institutes consisting of the University of Warwick, the University of Leicester, Queen's 
University Belfast, the University of Geneva, the Deutsches Zentrum für Luft- und Raumfahrt e.V. (DLR; under the 'Großinvestition GI-NGTS'), and the University of Cambridge, together with the UK Science and Technology Facilities Council (STFC; project reference ST/M001962/1). EG gratefully acknowledges support from the David and Claudia Harding Foundation in the form of a Winton Exoplanet Fellowship. PJW was supported by STFC consolidated grant ST/P000495/1. CAW acknowledges support by the STFC grant ST/P000312/1. This research has made use of the VizieR catalogue access tool, CDS, Strasbourg, France (DOI : $10.26093 / \mathrm{cds} /$ vizier). The original description of the VizieR service was published in A\&AS 143, 23(Ochsenbein, Bauer \& Marcout 2000). This research has made use of the SIMBAD database, operated at CDS, Strasbourg, France(Wenger et al. 2000). This work has made use of data from the European Space Agency (ESA) mission Gaia (https://www.cosmos.esa.int/gaia), processed by the Gaia Data Processing and Analysis Consortium (DPAC, https: //www.cosmos.esa.int/web/gaia/dpac/consortium). Funding for the DPAC has been provided by national institutions, in particular the institutions participating in the Gaia Multilateral Agreement. This publication makes use of data products from the Two Micron All Sky Survey, which is a joint project of the University of Massachusetts and the Infrared Processing and Analysis Center/California Institute of Technology, funded by the National Aeronautics and Space Administration and the National Science Foundation. Finally, we would like to thank the anonymous referee for their insightful reading of the manuscript and helpful suggestions for improvement.

\section{REFERENCES}

Aigrain S. et al., 2015, MNRAS, 450, 3211

Ambikasaran S., Foreman-Mackey D., Greengard L., Hogg D. W., O’Neil M., 2015, IEEE Trans. Pattern Anal. Mach. Intell., 38, 252

Angus R., Aigrain S., Foreman-Mackey D., McQuillan A., 2015, MNRAS, 450,1787

Angus R., Morton T., Aigrain S., Foreman-Mackey D., Rajpaul V., 2018, MNRAS, 474, 2094

Astropy Collaboration, 2013, A\&A, 558, A33

Astropy Collaboration, 2018, AJ, 156, 123

Balona L. A., Guzik J. A., Uytterhoeven K., Smith J. C., Tenenbaum P., Twicken J. D., 2011, MNRAS, 415, 3531

Barnes S. A., 2003, ApJ, 586, 464

Barnes J. R., Collier Cameron A., Donati J.-F., James D. J., Marsden S. C., Petit P., 2005, MNRAS, 357, L1

Bayliss D. et al., 2018, MNRAS, 475, 4467

Blanco V. M., 1949, PASP, 61, 183

Bouvier J., 2013, in Hennebelle P., Charbonnel C., eds, EAS Publ. Ser. Vol. 62, Role and Mechanisms of Angular Momentum Transport During the Formation and Early Evolution of Stars, EDP Sciences, 17 Avenue du Hoggar, Parc d'Activite de Courtabouf, BP 112, 91944 Les Ulis Cedex A, France. p. 143

Bouvier J., Matt S. P., Mohanty S., Scholz A., Stassun K. G., Zanni C., 2014, in Klessen R. S., Dullemond C. P., Henning T., eds, Protostars and Planets VI. University of Arizona Press, Tucson, AZ, p. 433

Brandt T. D., Huang C. X., 2015a, ApJ, 807, 24

Brandt T. D., Huang C. X., 2015b, ApJ, 807, 58

Cargile P. A., James D. J., Pepper J., Kuhn R. B., Siverd R., Stassun K. G., 2014, ApJ, 782, 29( C14)

Casewell S. L., Baker D. E. A., Jameson R. F., Hodgkin S. T., Dobbie P. D., Moraux E., 2012, MNRAS, 425, 3112

Chaboyer B., Demarque P., Pinsonneault M. H., 1995a, ApJ, 441, 865

Chaboyer B., Demarque P., Pinsonneault M. H., 1995b, ApJ, 441, 876

Charbonnel C., Decressin T., Amard L., Palacios A., Talon S., 2013, A\&A, $554, \mathrm{~A} 40$
Chazelas B. et al., 2012, in Stepp L. M., Gilmozzi R., Hall H. J., eds, Proc. SPIE Conf. Ser. Vol. 8444, Ground-Based and Airborne Telescopes IV. SPIE, Bellingham, p. 84440E

Choi J., Dotter A., Conroy C., Cantiello M., Paxton B., Johnson B. D., 2016, ApJ, 823, 102

Collier Cameron A., 2007, Astron. Nachr., 328, 1030

Covey K. R. et al., 2016a, ApJ, 822, 81

Covey K. R. et al., 2016b, ApJ, 822, 81

Daemgen S., Correia S., Petr-Gotzens M. G., 2012, A\&A, 540, A46

Daemgen S., Petr-Gotzens M. G., Correia S., Teixeira P. S., Brandner W., Kley W., Zinnecker H., 2013, A\&A, 554, A43

Davenport J. R. A., Hebb L., Hawley S. L., 2015, ApJ, 806, 212

de Epstein A. E. A., Epstein I., 1985, AJ, 90, 1211

Delorme P., Collier Cameron A., Hebb L., Rostron J., Lister T. A., Norton A. J., Pollacco D., West R. G., 2011, MNRAS, 413, 2218

Dotter A., 2016, ApJS, 222, 8

Douglas S. T., Agüeros M. A., Covey K. R., Kraus A., 2017, ApJ, 842, 83

Douglas S. T., Curtis J. L., Agüeros M. A., Cargile P. A., Brewer J. M., Meibom S., Jansen T., 2019, ApJ, 879, 100

Eggenberger P., Maeder A., Meynet G., 2005, A\&A, 440, L9

Epstein I., 1968, AJ, 73, 556

Feigelson E. D., Babu G. J., 2012, Modern Statistical Methods for Astronomy. Cambridge Univ. Press, Cambridge

Foreman-Mackey D., 2018, Res. Notes Am. Astron. Soc., 2, 31

Foreman-Mackey D., Barentsen G., 2019, https://doi.org/10.5281/zenodo.2 536576 (accessed January 2019)

Foreman-Mackey D., Hogg D. W., Lang D., Goodman J., 2013, PASP, 125, 306

Foreman-Mackey D., Agol E., Ambikasaran S., Angus R., 2017, AJ, 154, 220

Frasca A., Fröhlich H.-E., Bonanno A., Catanzaro G., Biazzo K., MolendaŻakowicz J., 2011, A\&A, 532, A81

Fröhlich H.-E., Frasca A., Catanzaro G., Bonanno A., Corsaro E., MolendaŻakowicz J., Klutsch A., Montes D., 2012, A\&A, 543, A146

Gaia Collaboration, 2018, A\&A, 616, A10( B18)

Gallet F., Bouvier J., 2013, A\&A, 556, A36

Gallet F., Bouvier J., 2015, A\&A, 577, A98

Gillen E. et al., 2017, A\&A, 599, A27

González J. F., Levato H., 2009, A\&A, 507, 541

Hartman J. D., Bakos G. Á., Kovács G., Noyes R. W., 2010, MNRAS, 408, 475

Herbst W., Bailer-Jones C. A. L., Mundt R., 2001, ApJ, 554, L197

Hoffman M. D., Gelman A., 2014, J. Mach. Learn. Res., 15, 1593

Irwin J., Aigrain S., Hodgkin S., Irwin M., Bouvier J., Clarke C., Hebb L., Moraux E., 2006, MNRAS, 370, 954

Irwin J., Hodgkin S., Aigrain S., Hebb L., Bouvier J., Clarke C., Moraux E., Bramich D. M., 2007, MNRAS, 377, 741

Johnstone C. P., Güdel M., Brott I., Lüftinger T., 2015, A\&A, 577, A28

Kaye A. B., Handler G., Krisciunas K., Poretti E., Zerbi F. M., 1999, PASP, 111,840

Lagarde N., Decressin T., Charbonnel C., Eggenberger P., Ekström S., Palacios A., 2012, A\&A, 543, A108

Larson R. B., 2003, Rep. Prog. Phys., 66, 1651

Leitzinger M., Odert P., Greimel R., Korhonen H., Guenther E. W., Hanslmeier A., Lammer H., Khodachenko M. L., 2014, MNRAS, 443, 898

McQuillan A., Mazeh T., Aigrain S., 2014, ApJS, 211, 24

Meibom S., Mathieu R. D., Stassun K. G., 2007, ApJ, 665, L155

Meibom S., Mathieu R. D., Stassun K. G., 2009, ApJ, 695, 679

Mermilliod J.-C., Platais I., James D. J., Grenon M., Cargile P. A., 2008, A\&A, 485, 95

Mermilliod J.-C., Mayor M., Udry S., 2009, A\&A, 498, 949

Micela G., Sciortino S., Favata F., Pallavicini R., Pye J., 1999, A\&A, 344, 83

Moraux E., Bouvier J., Stauffer J. R., Cuillandre J.-C., 2003, A\&A, 400, 891

Moraux E., Bouvier J., Stauffer J. R., Barrado y Navascués D., Cuillandre J.-C., 2007, A\&A, 471, 499 
Morin J. et al., 2008a, MNRAS, 384, 77

Morin J. et al., 2008b, MNRAS, 390, 567

Netopil M., Paunzen E., Heiter U., Soubiran C., 2016, A\&A, 585, A150

Nielsen M. B., Gizon L., Schunker H., Karoff C., 2013, A\&A, 557, L10

Patience J., Ghez A. M., Reid I. N., Matthews K., 2002, AJ, 123 , 1570

Pecaut M. J., Mamajek E. E., 2013, ApJS, 208, 9

Pillitteri I., Micela G., Sciortino S., Favata F., 2003, A\&A, 399, 919

Pillitteri I., Micela G., Sciortino S., Damiani F., Harnden F. R.,, Jr, 2004, A\&A, 421, 175

Pillitteri I., Micela G., Reale F., Sciortino S., 2005, A\&A, 430, 155

Platais I. et al., 2011, MNRAS, 413, 1024

Raghavan D. et al., 2010, ApJS, 190, 1

Rasmussen C. E., Williams C. K. I., 2006, Gaussian Processes for Machine Learning. MIT Press, Cambridge, MA

Rebull L. M. et al., 2016a, AJ, 152, 113

Rebull L. M. et al., 2016b, AJ, 152, 114

Rebull L. M., Stauffer J. R., Hillenbrand L. A., Cody A. M., Bouvier J., Soderblom D. R., Pinsonneault M., Hebb L., 2017, ApJ, 839, 92

Reiners A., Mohanty S., 2012, ApJ, 746, 43

Reinhold T., Reiners A., Basri G., 2013, A\&A, 560, A4

Ricker G. R. et al., 2014, in Oschmann J. M.,, Jr, Clampin M., Fazio G. G., MacEwen H. A., eds, Proc. SPIE Conf. Ser. Vol. 9143, Space Telescopes and Instrumentation 2014: Optical, Infrared, and Millimeter Wave. SPIE, Bellingham, p. 914320

Salvatier J., Wiecki T. V., Fonnesbeck C., 2016, Astrophysics Source Code Library, record ascl:1610.016

Stauffer J. R., Hartmann L. W., 1987, ApJ, 318, 337

Stauffer J. R., Hartmann L. W., Latham D. W., 1987, ApJ, 320, L51

Stauffer J. R. et al., 2010, ApJ, 719, 1859

Stauffer J. R. et al., 2016, AJ, 152, 115

Terndrup D. M., Stauffer J. R., Pinsonneault M. H., Sills A., Yuan Y., Jones

B. F., Fischer D., Krishnamurthi A., 2000, AJ, 119, 1303

The Theano Development Team, 2016, preprint (arXiv:1605.02688)

VanderPlas J. T., 2018, ApJS, 236, 16

Vidotto A. A. et al., 2014, MNRAS, 441, 2361

West R. G. et al., 2019, MNRAS, 486, 5094

Westerlund B. E., 1963, MNRAS, 127, 83

Westerlund B. E., Garnier R., Lundgren K., Pettersson B., Breysacher J., 1988, A\&AS, 76, 101

Wheatley P. J. et al., 2018, MNRAS, 475, 4476

Wilson O. C., 1966, ApJ, 144, 695

Ochsenbein F., Bauer P., Marcout J., 2000, A\&AS, 143, 23

Wenger M. et al., 2000, AAPS, 143, 9

\section{SUPPORTING INFORMATION}

Supplementary data are available at MNRAS online.

Table 1. Identification, photometric, multiplicity, and period information for periodic Blanco 1 stars.

Table 2. Identification, photometric, and multiplicity information for Blanco 1 stars without a detected period.

Please note: Oxford University Press is not responsible for the content or functionality of any supporting materials supplied by the authors. Any queries (other than missing material) should be directed to the corresponding author for the article.

\section{APPENDIX A: REMOVING RESIDUAL MOON VARIATIONS ARISING FROM INCOMPLETE BACKGROUND CORRECTION}

During the course of the 200-d NGTS observations, the moon passes through several lunar cycles, with corresponding brightness variations. These are corrected for within the standard NGTS pipeline through a SYSREM-based detrending algorithm (see Wheatley et al. 2018 , for more details). This works well for all but the faintest stars (NGTS $\gtrsim 15 \mathrm{mag}$ ), which typically still display a level of variability in phase with the lunar cycle. We define moon signal here as periodic variability between $25 \mathrm{~d}<P<30 \mathrm{~d}$, whose phase shape coincided with the moon's brightness variations during each lunar cycle. In some cases, these variations have an amplitude comparable to the rotation signals we are trying to detect. We opted therefore to remove the residual moon signal, where applicable, during our procedure for estimating rotation periods. The procedure is described below.

For each light curve, we performed an initial LS fit. If moon signal was detected in the four strongest non-aliased peaks, it was removed. The removal process comprised two steps. The light curve was folded on the detected 'moon' period and detrended for the dominant variation pattern using a Savitzky-Golay (SG) filter followed by a convolution. We opted to perform this two-step process as it allowed us to best capture the shape of the moon signal on the first pass (SG filter) and then smooth this signal (via convolution) to apply a smooth detrending of the moon signal.

In practice, the residual moon signal was only apparent in stars fainter than $\sim 14.5$ mag in the NGTS band, which corresponds to early M stars and later spectral types in Blanco 1. We checked that removing the moon signal did not significantly change the rotation periods determined for a handful of example light curves, with signals across a range of amplitudes. For some low-mass stars, however, it allowed us to determine rotation periods with a higher degree of confidence.

\footnotetext{
${ }^{1}$ Astrophysics Group, Cavendish Laboratory, J. J. Thomson Avenue, Cambridge $\mathrm{CB} 3 \mathrm{OHE}, \mathrm{UK}$

${ }^{2}$ Institute of Astronomy, University of Cambridge, Madingley Rise, Cambridge CB3 OHA, UK

${ }^{3}$ Center for Computational Astrophysics, Flatiron Institute, New York, NY 10010, USA

${ }^{4}$ Department of Physics, University of Warwick, Gibbet Hill Road, Coventry CV4 7AL, UK

${ }^{5}$ Centre for Exoplanets and Habitability, University of Warwick, Gibbet Hill Road, Coventry CV4 7AL, UK

${ }^{6}$ Department of Physics and Astronomy, University of Leicester, Leicester LE1 7RH, UK

${ }^{7}$ Astrophysics Research Centre, School of Mathematics and Physics, Queen's University Belfast, Belfast BT7 1NN, UK

${ }^{8}$ Departamento de Astronomia, Universidad de Chile, Casilla 36-D, Santiago, Chile

${ }^{9}$ Centro de Astrofísica y Tecnologías Afines (CATA), Casilla 36-D, Santiago, Chile

${ }^{10}$ Institute of Planetary Research, German Aerospace Center, Rutherfordstr 2, D-12489 Berlin, Germany
}

This paper has been typeset from a $\mathrm{T}_{\mathrm{E}} \mathrm{X} / \mathrm{LT} \mathrm{E} \mathrm{X}$ file prepared by the author. 